\title{
Large-eddy simulation of the up-slope boundary layer
}

\author{
By ULRICH SCHUMANN \\ DLR, Institute of Atmospheric Physics, D-8031 Oberpfaffenhofen, Federal Republic of Germany
}

(Received 13 June 1989; revised 24 October 1989)

\section{SUMMARY}

Large-eddy simulation (LES) is used to determine the turbulent structure of the steady-state up-slope boundary layer (UBL) which forms at a uniformly heated inclined or vertical plane surface below a stratified atmosphere at rest. At proper scales, the problem depends solely on the slope angle $\alpha$ and the surface roughness height $z_{0}$ relative to a length scale $H$ which is a function of stratification and surface heating. We cover $2^{\circ} \leqslant \alpha \leqslant 90^{\circ}$ and $3 \times 10^{-5} \leqslant z_{0} / H \leqslant 3 \times 10^{-2}$ by a series of LESs. Simulations using different grids show that the results are only weakly sensitive to truncation errors. The UBL approaches a steady-state mean-profile by slowly decaying oscillations; its frequency equals the Brunt-Väisälä frequency times $\sin \alpha$. In the LES, convergence towards steady state has been enforced by adjusting the fields according to integral steady-state conditions. For $\alpha \leqslant 10^{\circ}$, a well-mixed layer is formed which causes a strong temperature inversion and strong down-slope flow at the outer edge of the UBL. Large-scale coherence is weak with some indications of crossslope rolls for small inclination angles and longitudinal rolls for steep slopes. Several mean quantities have been tabulated as a function of slope angle and surface roughness and approximating power laws are given. The dependence of mean quantities on the slope angle is difficult to explain with simple models, but the influence of surface roughness closely follows earlier results.

\section{INTRODUCTION}

We consider the turbulent boundary layer over an unbounded homogeneous rough plane, inclined to the horizontal plane by an angle $\alpha$. The free atmosphere above the surface is at rest and uniformly stratified. This study is restricted to nonrotating systems and to the day-time case. We assume that the atmosphere is heated solely from the surface at a constant and uniform heat flux rate. The resultant buoyancy induces a narrow 'up-slope boundary layer (UBL)', also called 'buoyancy layer' (Veronis 1967, Turner 1973 , p. 243) or 'anabatic wind' (see Whiteman 1990). After some time, the flow approaches steady state with mean values depending solely on the coordinate normal to the surface. For laminar flow, this problem has been solved by Prandtl (1942), (see Fig. 1). Actually, Prandtl considered the case of prescribed temperature difference $\Delta T_{\mathrm{s}}$, but the laminar solution applies equally to prescribed surface heat flux.

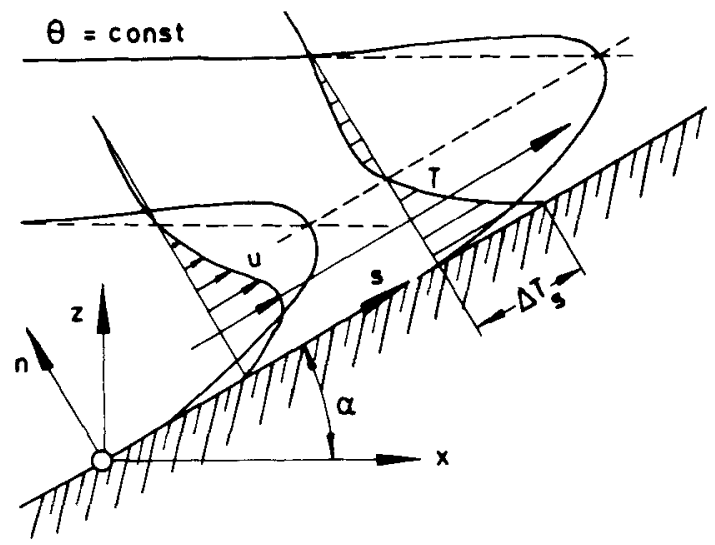

Figure 1. The velocity and temperature anomaly profiles for an up-slope boundary layer at a slope with angle $\alpha$ in a linearly stratified environment. The curves represent Prandtl's exact solutions for laminar flow. 
Real atmospheric slope layers are rarely uniform. They are spatially and temporally variable and appear often highly intermittent in structure. Such slope flows are subject to cross-slope valley winds (Hennemuth and Schmidt 1985) or variations of the slope angle, variable atmospheric stratification and local surface inhomogeneities (Vergeiner and Dreiseitl 1987). Nevertheless, understanding of the idealized UBL is of importance for many applications in mountain meteorology, e.g., the net heating from the slope's surface is partially transferred to the valley's atmosphere by sinking motion in the core of the valley for continuity (Vergeiner 1982). Knowledge of the up-slope mass flux is required for analysis of pollution transport (Yamada and Bunker 1988). Since the temperature difference at the surface depends strongly on the surface heat flux, one can deduce the heat flux from remote measurements of the surface temperature, provided all the other important parameters can be estimated to sufficient accuracy (Mannstein 1989). The relationship between surface fluxes and integrals of the mean profiles are required to parametrize the variation of bulk properties of the boundary layer without taking account of its internal structure (Manins and Sawford 1979).

Moreover, it is of interest to explore the structure of turbulent motions in the UBL because this information is needed for turbulence modelling. In particular, one needs to know the turbulence variances and scales and whether buoyancy or shear causes coherent flow patterns like cellular convection or roll vortices. Such patterns are to be expected because linear theories for similar flows (Brown 1980) show that velocity profiles with an inflection point are inherently unstable and that for convection in the presence of shear the longitudinal roll mode is dominant. Gill and Davey (1969) studied the stability of the laminar boundary layer regime on a vertical wall with respect to two-dimensional disturbances which are invariant in the cross-slope direction. They showed that buoyancydriven instability (as distinct from inflection point instability) causes the growth of disturbances which may have the form of waves travelling faster than the flow. A complete three-dimensional stability analysis does not seem to exist for the UBL, but Clever and Busse (1977) show for an inclined layer of finite thickness heated from below that longitudinal rolls, which form first, exhibit a wavy instability at steep slopes. In the fully turbulent state, convective turbulence may destroy shear-induced longitudinal rolls when the boundary layer is thick in comparison to the Obukhov length (Deardorff 1972).

Prandtl's (1942) theory predicts a linear relationship between up-slope wind speed, surface temperature difference and heat flux for steady laminar flow. The depth of the laminar boundary layer scales with $(\sin \alpha)^{-1 / 2}$. It will take a very long time before a UBL reaches steady state at small angles because of its great thickness. Defant (1949) has applied Prandtl's laminar theory to atmospheric cases by adjusting the constant diffusivities so that the results match observations. Other models consider turbulent boundary layers in terms of their mean properties using 'hydraulic models' but these models have been applied mainly to katabatic winds over cooled surfaces (e.g. see Kondo and Sato 1988). The most difficult parameter yet to be established for the UBL is its depth; Prandtl's prediction applied only to constant diffusivities. One-dimensional models which compute the local turbulence transport have been set up by Lykosov and Gutman (1972), Rao and Snodgrass (1981) and Brehm (1986). In the two latter papers, the turbulent fluxes are determined with a transport equation for the kinetic energy of turbulent fluctuations and prescribed mixing lengths (Mellor and Yamada 1982). Brehm (1986) considered the UBL while Rao and Snodgrass (1981) discussed down-slope winds. Brehm has applied the results of his model for $2^{\circ} \leqslant \alpha \leqslant 30^{\circ}$ to set up parametrizations which relate various mean quantities as a function of inclination angle and an equivalent Rossby number as suggested by Gill (1966) and Veronis (1967). The UBL at a vertical wall has been analysed for laminar flow by Gill (1966). Laminar flows induced at a slope by a 
spatially finite disturbance have been considered by Egger (1981). Various model concepts and their application to valley flows are discussed by Egger (1989). No theory has as yet resolved the three-dimensional and time-dependent structure of the turbulence within the UBL.

Day-time measurements of up-slope winds at Alpine slopes and elsewhere have been summarized by Defant (1949), Atkinson (1981), Hennemuth and Schmidt (1985), Brehm (1986) and Whiteman (1990). The data for slope-angles between $10^{\circ}$ and $45^{\circ}$ show a velocity boundary layer which extends typically over a thickness of 60 to $140 \mathrm{~m}$. The maximum up-slope velocity amounts to values between 1.2 and $4 \mathrm{~m} \mathrm{~s}^{-1}$. The temperature measured close to the surface exceeds the outside temperature at the same height by up to $7 \mathrm{~K}$. The thermal boundary layer is roughly half as thick as the velocity boundary layer. The results agree qualitatively with Prandtl's theory if one adjusts the turbulent diffusivities accordingly (Defant 1949); but the velocity maximum is reached closer to the surface. The data are, however, insufficient to determine the quantitative relationships between the profiles, the surface heat flux, the surface roughness and the stratification. Turbulence measurements have been reported for the nocturnal slope flow (Horst and Doran 1988, Whiteman 1989) but do not exist for an atmospheric UBL. Mean profile and turbulence measurements are available also for a shallow baroclinic convective boundary layer over a horizontal surface from Zhou et al. (1985), where inclination of isotherms has an effect similar to that of an inclined surface in the barotropic atmosphere which we are considering. Zhou et al. (1985) estimated the horizontal temperature gradient between $0 \cdot 12$ and $0 \cdot 18 \mathrm{~K} \mathrm{~km}^{-1}$ but have not measured the vertical gradient of potential temperature above the boundary layer. Assuming typical values for such a gradient (e.g. $3 \mathrm{~K} \mathrm{~km}^{-1}$ ) implies an inclination angle of the isotherms in this experiment of about a few degrees.

In a laboratory experiment, Deardorff and Willis (1987) investigated convection in a tank of water containing a UBL for $\alpha=10^{\circ}$. They report on turbulence statistics in addition to mean profiles and find significant contributions both from buoyancy and shear to turbulence production. There was no significant tendency for the shear or inflection point of the mean velocity profile to promote any organization in convection cell patterns or to cause roll vortices. However, the finite up-slope length of the tank and the depth of the water layer caused strong up-slope gradients of the simulated slope layer with variable thickness of the mixed layer and baroclinicity above and, hence, the experiment only approximates the properties of a uniform UBL.

This paper presents results from a large-eddy simulation (LES) of the UBL for angles $\alpha$ between 2 and $90^{\circ}$. LES was applied successfully to determine the turbulent structure in convective boundary layers over horizontal planes as demonstrated by Deardorff (1972) and Schmidt and Schumann (1989). For the present purpose, the method of Schmidt and Schumann (1989) is extended to sloping surfaces.

\section{Problem formulation AND SOlUtion METHOD}

We consider a plane inclined by the slope-angle $\alpha$ as sketched in Fig. 1. The atmosphere above the plane is approximated in the Boussinesq sense, i.e. density $\rho$ is assumed to be constant except for buoyancy effects. The vertical gradient of potential temperature $d \theta / d z$, and the volumetric expansion coefficient $\beta=-(\partial \rho / \partial \theta) / \rho$ are assumed to be constant. With gravity $g$ we form the Brunt-Väisälä frequency $N=$ $(g \beta d \theta / d z)^{1 / 2}$. The plane has a rough surface which is characterized by a roughness height $z_{0}$. This is certainly a very crude approach for characterizing real surfaces, which limits the validity of the present analysis, but we have no better tool at hand. From the 
surface, heat enters the atmosphere with a constant prescribed kinematic heat flux $Q_{\mathrm{s}}$ ('temperature flux') per unit surface area. Coriolis forces are not included in the analysis. The parameters can be used to define a characteristic velocity scale $v_{*}$, height scale $H$, and temperature scale $\theta_{*}$ :

$$
v_{*}=\left(\beta g Q_{\mathrm{s}} / N\right)^{1 / 2} \quad H=v_{*} / N \quad \theta_{*}=Q_{\mathrm{s}} / v_{*} .
$$

Note that the selected scales differ from the usual convective and surface-layer scales (Deardorff 1972) which depend on the boundary layer thickness and the surface momentum flux; these are internal parameters not known a priori. The length scale $H$ is not the boundary-layer thickness which, as we will see, depends on $H$ and the slope angle $\alpha$. For small molecular diffusivities, the problem is completely characterized by $\alpha$, the height ratio $H / z_{0}$, and the non-dimensional time $t N$.

For atmospheric cases, realistic values of the independent parameters are, e.g.,

$$
\begin{gathered}
d \theta / d z=0.003 \mathrm{~K} \mathrm{~m}^{-1} \quad \beta=300^{-1} \mathrm{~K}^{-1} \\
g \cong 10 \mathrm{~m} \mathrm{~s}^{-2} \quad Q_{s}=0.1 \mathrm{Km} \mathrm{s}^{-1} \quad z_{0}=0.2 \mathrm{~m} .
\end{gathered}
$$

The derived quantities are

$$
N=0.01 \mathrm{~s}^{-1} \quad v_{*}=0.58 \mathrm{~m} \mathrm{~s}^{-1} \quad H=58 \mathrm{~m} \quad \theta_{*}=0.17 \mathrm{~K} .
$$

We use a Cartesian frame of reference $x_{i}=(s, y, n)$ (see Fig. 1) aligned with the slope. The corresponding velocity components are $u_{i}=(u, v, w)$. In the atmosphere at rest, the gradient of hydrostatic pressure balances the mean buoyancy forces. Temperature and pressure deviations from this basic state are denoted by $T$ and $p$, respectively. The splitting of temperature into a basic state and turbulent fluctuations is essential for this study because it allows us to apply periodic boundary conditions at the lateral boundaries for all fluctuating fields. The balance equations, written in Einstein's summation notation, are

$$
\begin{gathered}
\frac{\partial \bar{u}_{j}}{\partial x_{j}}=0 \\
\frac{\partial \bar{u}_{i}}{\partial t}+\frac{\partial\left(\bar{u}_{j} \bar{u}_{i}\right)}{\partial x_{j}}=-\frac{1}{\rho} \frac{\partial \bar{p}}{\partial x_{i}}-\frac{\partial}{\partial x_{j}}\left(\overline{u_{i}^{\prime \prime} u_{j}^{\prime \prime}}\right)+\beta g_{i} \bar{T} \\
\frac{\partial \bar{T}}{\partial t}+\frac{\partial\left(\bar{u}_{j} \bar{T}\right)}{\partial x_{j}}+\bar{u}_{j} \frac{d \theta}{d x_{j}}=-\frac{\partial}{\partial x_{j}}\left(\overline{u_{j}^{\prime \prime} T^{\prime \prime}}\right) .
\end{gathered}
$$

In the inclined frame of reference the gravity components and the mean temperature gradient are given by

$$
g_{i}=(\sin \alpha, 0, \cos \alpha) g \quad \frac{d \theta}{d x_{i}}=(\sin \alpha, 0, \cos \alpha) \frac{d \theta}{d z} .
$$

Bars denote grid-volume averages. Subgrid motions, denoted by double-primes, induce fluxes which require modelling. We use a subgrid-scale (SGS) model which integrates a closed transport equation for SGS kinetic energy $\overline{E^{\prime \prime}} \equiv \vec{u}_{i}^{\prime \prime 2} / 2$, including buoyancy fluxes in $s$ and $n$ directions:

$$
\frac{D \overline{E^{\prime \prime}}}{D t}=-\overline{u_{i}^{\prime \prime} u_{j}^{\prime \prime}} \frac{\partial \bar{u}_{i}}{\partial x_{j}}+\beta g_{i} \overline{u_{i}^{\prime \prime} T^{\prime \prime}}+\frac{\partial}{\partial x_{i}}\left(\frac{5}{3} \ell c_{3 m} \overline{E^{\prime \prime}} 1 / 2 \frac{\partial \overline{E^{\prime \prime}}}{\partial x_{i}}\right)-c_{\varepsilon m} \frac{\overline{E^{\prime \prime}} / 2}{\ell} .
$$

The SGS fluxes are determined from the gradients of resolved fields

$$
\overline{u_{i}^{\prime \prime} u_{j}^{\prime \prime}}=-\nu_{i}\left(\frac{\partial \bar{u}_{i}}{\partial x_{j}}+\frac{\partial \bar{u}_{j}}{\partial x_{i}}\right)+\frac{1}{3} \delta_{i j} \overline{u_{k}^{\prime 2}} \quad \overline{u_{i}^{\prime \prime} T^{\prime \prime}}=-\gamma_{t}\left(\frac{\partial \bar{T}}{\partial x_{i}}+\frac{d \theta}{d x_{i}}\right)
$$


with the Prandtl-Kolmogorov diffusivities

$$
\nu_{t}=c_{\nu} \ell \overline{E^{\prime \prime}} 1 / 2 \quad \gamma_{t}=c_{\gamma} \ell{\overline{E^{\prime \prime}}}^{1 / 2}
$$

and with a length scale $\ell$ prescribed as a function of distance $n$ from the surface and of the mesh spacing $\Delta$ (we use equal spacings in the three directions) by

$$
\ell=\min \left(\Delta, c_{\ell} n\right) \text {. }
$$

The model coefficients are the same as those deduced in Schmidt and Schumann (1989):

$$
c_{\nu}=0.0856 \quad c_{\gamma}=0.204 \quad c_{3 m}=0.2 \quad c_{\varepsilon m}=0.845 \quad c_{\ell}=c_{\varepsilon m} .
$$

The present model is simplified, however, in that the buoyancy contributions to the anisotropic parts of the fluxes described by Schmidt and Schumann (1989) are neglected, because the coupling with vertical and horizontal buoyancy fluxes would make the scheme unduly complicated. This simplification is reasonable if the resolution is fine enough. It will be checked later by comparing results from simulations with different resolutions.

The numerical integration scheme is described in Schumann et al. (1987). It employs an equidistant staggered grid and finite difference approximations. The momentum and continuity equations are approximated by second-order central differences in space with the Adams-Bashforth scheme for integration in time. The balance equations for temperature and for SGS kinetic energy are approximated by the second-order up-wind scheme of Smolarkiewicz (1984).

At the outer boundary of the computational domain, free-slip boundary conditions are used. A radiative condition relates horizontal Fourier transforms of pressure with the vertical velocity and an appropriate Brunt-Väisälä frequency $N_{\mathrm{b}}$ as proposed by Bougeault (1983) and Klemp and Durran (1983) for hydrostatic horizontal flows,

$$
\hat{p}=N_{\mathrm{b}} \rho \hat{w} / k \quad N_{\mathrm{b}}^{2}=\beta g(d \theta / d z) \cos \left(\min \left(\alpha, 30^{\circ}\right)\right) .
$$

The 'hat' denotes the Fourier modes corresponding to the magnitude $k=\left(k_{s}^{2}+k_{y}^{2}\right)^{1 / 2}$ of the wavenumbers in the outer $s-y$-plane. This boundary condition has not been designed for sloping flows but has been found to give reasonable results in this study: the minimum function is introduced based on heuristic reasoning to prevent energy accumulating near the outer boundary when the slope-angles are large. At the surface, the temperature flux $Q_{s}$ determines the SGS flux, and the normal fluxes of horizontal momentum are evaluated from the Monin-Obukhov relationships, as explained in Schmidt and Schumann (1989), for given surface roughness length $z_{0}$. The surface temperature is also deduced from these relationships. For simplicity, we assume that the same value of $z_{0}$ applies to both momentum and heat transfer. The 'surface temperature' is defined as $T\left(z_{0}\right)$.

Details of the initial conditions do not affect the final solutions but some initial disturbances are necessary because otherwise the code would predict a laminar flow. The initial values prescribe zero mean values of $u_{i}$ and $T$ with random numbers imposed on all velocity components and the temperature having the (normalized) magnitude 0.25 $\exp (-n / d)$. The effective thickness of the boundary layer is estimated for this purpose as $d=0 \cdot 1 \mathrm{H} / \mathrm{sin} \alpha$ from preliminary tests with the present code. The resultant velocity field is adjusted for continuity and the kinematic wall boundary condition by applying a pressure correction as described in Schumann et al. (1987). The SGS kinetic energy is initialized by $E=0.5 v_{*}^{2} \exp (-2 n / d)$.

Further parameters of the numerical scheme are the size $X . Y . Z$ of the computational domain, the corresponding number of grid points $N_{x} \cdot N_{y} \cdot N_{z}$, the maximum simulation time $t_{\max }$, and the time-step $\Delta t$. These parameters are given in Table 1 for the various cases discussed in this paper. Cases Bxx are coarse-grid simulations; $x x$ denotes the value 
TABLE 1. PHYSICAL AND NUMERICAL PARAMETERS

\begin{tabular}{lrlllllllll}
\hline Case & $\alpha$ & $z_{\mathrm{o}} / H$ & \multicolumn{1}{c}{$X / H$} & $Y / H$ & $Z / H$ & $t_{\max } N$ & $N_{x}$ & $N_{y}$ & $N_{z}$ & $\Delta t N$ \\
\hline B02 & 2 & $0 \cdot 003$ & 64 & 32 & 24 & 600 & 64 & 32 & 24 & $0 \cdot 05$ \\
B04 & 4 & $0 \cdot 003$ & $42 \cdot 67$ & $21 \cdot 33$ & 16 & 240 & 64 & 32 & 24 & $0 \cdot 04$ \\
B07 & 7 & $0 \cdot 003$ & 32 & 16 & 12 & 240 & 64 & 32 & 24 & $0 \cdot 04$ \\
B10 & 10 & $0 \cdot 003$ & $25 \cdot 6$ & $12 \cdot 8$ & $9 \cdot 6$ & 160 & 64 & 32 & 24 & $0 \cdot 04$ \\
B20 & 20 & $0 \cdot 003$ & 16 & 8 & 6 & 100 & 64 & 32 & 24 & $0 \cdot 025$ \\
B30 & 30 & $0 \cdot 003$ & 12 & 6 & $4 \cdot 5$ & 60 & 64 & 32 & 24 & $0 \cdot 015$ \\
B45 & 45 & $0 \cdot 003$ & 8 & 4 & 3 & 40 & 64 & 32 & 24 & $0 \cdot 01$ \\
B90 & 90 & $0 \cdot 003$ & $4 \cdot 8$ & $2 \cdot 4$ & $1 \cdot 8$ & 20 & 64 & 32 & 24 & $0 \cdot 005$ \\
R02 & 2 & $0 \cdot 0015$ & 64 & 32 & 24 & 600 & 64 & 32 & 24 & $0 \cdot 05$ \\
R10 & 10 & $0 \cdot 0015$ & $25 \cdot 6$ & $12 \cdot 8$ & $9 \cdot 6$ & 160 & 64 & 32 & 24 & $0 \cdot 04$ \\
R30 & 30 & $0 \cdot 0015$ & 12 & 6 & $4 \cdot 5$ & 60 & 64 & 32 & 24 & $0 \cdot 015$ \\
R45 & 45 & $0 \cdot 0015$ & 8 & 4 & 3 & 40 & 64 & 32 & 24 & $0 \cdot 01$ \\
R90 & 90 & $0 \cdot 0015$ & $4 \cdot 8$ & $2 \cdot 4$ & $1 \cdot 8$ & 20 & 64 & 32 & 24 & $0 \cdot 005$ \\
C10 & 10 & $3 \times 10^{-2}$ & $25 \cdot 6$ & $12 \cdot 8$ & $0 \cdot 6$ & 100 & 64 & 32 & 24 & $0 \cdot 04$ \\
E10 & 10 & $3 \times 10^{-4}$ & $25 \cdot 6$ & $12 \cdot 8$ & $0 \cdot 6$ & 100 & 64 & 32 & 24 & $0 \cdot 04$ \\
F10 & 10 & $3 \times 10^{-5}$ & $25 \cdot 6$ & $12 \cdot 8$ & $0 \cdot 6$ & 100 & 64 & 32 & 24 & $0 \cdot 04$ \\
D10 & 10 & $0 \cdot 003$ & $25 \cdot 6$ & $12 \cdot 8$ & $9 \cdot 6$ & 60 & 128 & 64 & 48 & $0 \cdot 015$ \\
D90 & 90 & $0 \cdot 003$ & $4 \cdot 8$ & $2 \cdot 4$ & $1 \cdot 8$ & 20 & 128 & 64 & 48 & $0 \cdot 0025$ \\
\hline
\end{tabular}

( $\alpha=$ slope angle, $z_{0}=$ roughness height, $X, Y, Z=$ size of the computational domain, $t_{\max }=$ maximum integration time, $N_{x}, N_{y}, N_{z}=$ number of mesh points in the three directions, $\Delta t=$ time step, $H=$ height scale, $N=$ Brunt-Väisälä frequency.)

of the slope-angle. B10 requires 72 min computing time on a CRAY-XMP. The resolution in case D10 is double that in case $\mathrm{B} 10$ and requires $7 \cdot 6$ hours computer time. The roughness parameter equals $z_{0} / H=0.003$ in cases Bxx and 0.0015 in cases Rxx; stronger variations are considered in cases $\mathrm{C} 10, \mathrm{E} 10$ and $\mathrm{F} 10$. The size of the computational domain is selected so that it extends over at least two and at most three boundarylayer thicknesses in the normal direction. The length $X$ is taken to be twice the value of $Y$ because we expect up-slope oriented rolls. The lateral domain sizes should be big enough to permit the development of large-scale coherent structures. The maximum integration time is a compromise between computational effort and the desire to reach a quasi-steady state. Its value is taken large enough to allow the fluid near the wall to travel at least once over the distance $X$, i.e. the upstream extent of the computational domain. Moreover, it is large in comparison to the time-period $2 \pi /(N \sin \alpha)$ of gravity oscillations (as explained below). The latter criterion is particularly demanding at small slope-angles. The time-step is determined from a linear stability analysis.

In steady state, the bulk properties of the UBL

$$
\begin{gathered}
U D=\int_{0}^{\infty} u d n \quad W D=\int_{0}^{\infty} w d n \quad T D=\int_{0}^{\infty} T d n \\
U U D=\int_{0}^{\infty}\langle u\rangle^{2} d n \quad U T D=\int_{0}^{\infty}\langle u\rangle\langle T\rangle d n
\end{gathered}
$$

satisfy the following integral relationships:

$$
\begin{gathered}
\frac{\partial U D}{\partial t}+\frac{\partial U U D}{\partial s}+\frac{\partial}{\partial s} \int_{0}^{\infty}\left\langle u^{\prime 2}\right\rangle d n=\left\langle u^{\prime} w^{\prime}\right\rangle_{\mathrm{s}}+\beta g T D \sin \alpha=0 \\
\frac{\partial T D}{\partial t}+\frac{\partial U T D}{\partial s}+\frac{\partial}{\partial s} \int_{0}^{\infty}\left\langle u^{\prime} T^{\prime}\right\rangle d n+W D \frac{d \theta}{d z} \cos \alpha=Q_{\mathrm{s}}-U D \frac{d \theta}{d z} \sin \alpha=0 .
\end{gathered}
$$


The brackets $\langle>$ denote ensemble means which we will compute by averaging over planes parallel to the surface. Single primes denote deviations from these mean values. The right-hand side of the equation for $U D$ states a balance between surface friction $\left\langle u^{\prime} w^{\prime}\right\rangle=-u_{*}^{2}$ and integrated up-slope buoyancy. It shows that the mean temperature excess in the boundary layer is controlled by the external parameter $\beta g \sin \alpha$ and the surface friction. The second equation represents the balance of surface heating $Q_{\mathrm{s}}=$ $\left\langle w^{\prime} T^{\prime}\right\rangle_{s}$ and up-slope advection of heat against the mean temperature gradient. It shows that the mean up-slope mass flux is totally controlled by external parameters for given surface heat flux, and this fact has been verified experimentally by Hennemuth and Schmidt (1985).

As we will show later, it requires very long integration times to approach a state where these relationships are satisfied. Therefore we employ a 'control scheme,' which reduces the velocity and temperature field after each time-step if $U D$ or $T D$ is larger than the corresponding steady-state values. The reduction is performed by multiplying the horizontal velocity and the temperature fields with a relaxation factor $r$, which depends on the ratio $R$ of the steady-state value of $U D$ or $T D$ relative to their actual value, as $r=1+0 \cdot 1(R-1)$, until the steady-state conditions are satisfied within $2 \%$. Hence, the effect of this scheme vanishes in the steady state.

\section{RESULTS}

(a) Approach towards steady state

Figure 2 depicts the temporal development of a few mean properties versus time. The thick curve applies to case $\mathrm{B} 10^{*}$ which is run without control of steady-state conditions. We observe that the surface temperature difference $\Delta T_{\mathrm{s}}$ approaches steady state rather quickly. However, the maximum of the horizontally averaged velocity shows quite large oscillations which are damped only a little. $U D$ oscillates between zero and about twice the steady-state value, which we know precisely from Eq. (15), and TD

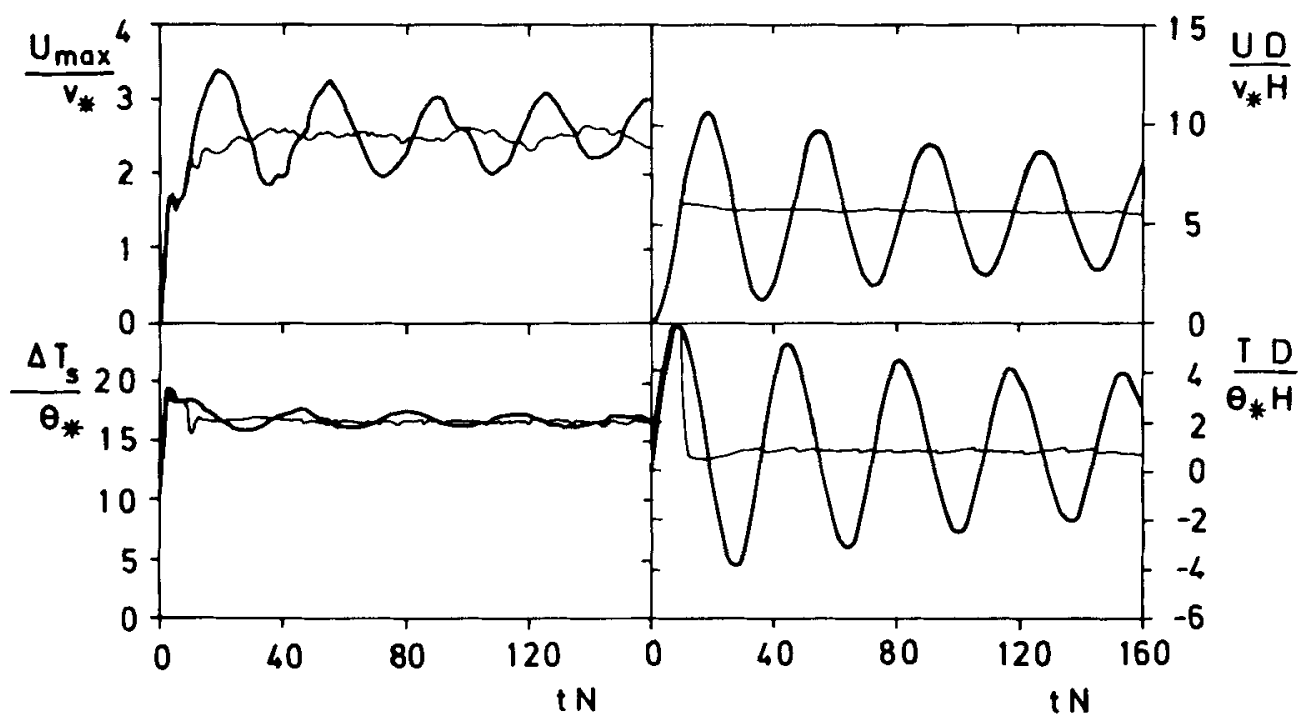

Figure 2. Transient development towards steady state in case $\mathrm{B} 10^{*}$ without control of integral balances (thick curves) and in case B10 with control (thin curves) in terms of maximum mean up-slope velocity $U_{\max }$, surface temperature difference $\Delta T_{\mathrm{s}}$, integral mass flow rate $U D=\int\langle u(n)\rangle d n$, and integral temperature deviation $T D=$ $\int\langle T(n)\rangle d n$ 
oscillates even more strongly between large negative and positive values. Such oscillations were to be expected, as can be seen by combining Eqs. (14) and (15) for unsteady homogeneous flows, in which $\partial / \partial s=0$,

$$
\begin{gathered}
\frac{\partial^{2} T D}{\partial t^{2}}+N^{2} \sin ^{2} \alpha T D=u_{*}^{2} \frac{d \theta}{d z} \sin \alpha \\
\frac{\partial^{2} U D}{\partial t^{2}}+N^{2} \sin ^{2} \alpha U D=\beta g Q_{\mathrm{s}} \sin \alpha-\frac{\partial}{\partial t} u_{*}^{2} .
\end{gathered}
$$

Thus the values of $T D$ and $U D$ should oscillate with a time-period

$$
2 \pi /(N \sin \alpha)=36 \cdot 2 / N \text { for } \alpha=10^{\circ}
$$

and this is in precise agreement with the numerical results. In fact, Schlieren pictures displayed in Fig. 2.7 of Turner (1973) together with proper analysis (Turner 1973, p. 23) show that the relationship between inclined oscillations and the given frequency exists in a stratified medium even without a surface. Obviously, the damping due to surface friction is weak. Also energy loss due to radiating gravity waves cannot damp this type of oscillation. For $\alpha=2^{\circ}$ the oscillations have a time period of $180 / N$ which amounts typically to five hours. Hence, steady state will be reached in nature only for large slopeangles.

The thin curves in Fig. 2 show the results which we obtain using the control scheme described in the previous section. This scheme is quite successful in forcing the flow towards steady state although some small fluctuations remain. In addition a test case was run, in which the control scheme was used until $t N=160$, but then the run was continued until $t N=240$ without this scheme. The results confirmed that the solution stays close to steady without significant changes in the mean values but with slight increase in the standard deviations.

In Table 2, we have summarized resultant mean values for all cases. The table contains also the standard deviations as computed from the last sixth of the simulation period. We see that the standard deviations are very small for quantities which are closely related to wall friction while quantities like $U U D$ or $U T D$, which depend on the form of the resultant profiles, show larger but still acceptable deviations. Comparison of the results for case B10 (with steady-state control) and case B10* (without control) shows that the mean values are not changed significantly by the control scheme. Comparison of the cases with fine resolution, D10 and D90, with those from the coarse-grid simulations, B10 and B90, shows that the coarse-grid results are close to the fine-grid results and hence only weakly affected by truncation or SGS-model errors. The remaining differences are larger for $\alpha=90^{\circ}$ than for $\alpha=10^{\circ}$ because of the relatively small boundary-layer thickness at the vertical surface.

\section{(b) Mean profiles}

Mean profiles are evaluated by averaging the resultant fields over planes parallel to the surface. These mean profiles are denoted by angle brackets, e.g. $\langle u\rangle$, and are functions of the normal coordinate $n$. Figure 3 shows, for example, the mean velocity and mean temperature profiles, for various slope-angles $\alpha \leqslant 30^{\circ}$. The error bars represent the standard deviations of the profiles from their mean values in the second half of the integration period. Differences in the results for coarse and fine grids are insignificant in comparison to these standard deviations. The profiles show qualitatively the same form as the laminar result depicted in Fig. 1 but with significant quantitative differences, in particular for small slope-angles $\left(\alpha \leqslant 10^{\circ}\right)$. The mean temperature in the first grid cell is 
TABLE 2. MEAN VALUE RESULTS FOR STEADY STATE INCLUDING STANDARD DEVIATIONS

\begin{tabular}{|c|c|c|c|c|c|c|c|c|c|c|}
\hline $\begin{array}{l}\text { var. } \\
\text { unit }\end{array}$ & $\begin{array}{l}u_{*} \\
v_{*}\end{array}$ & $\begin{array}{c}U_{\max } \\
v_{*}\end{array}$ & $\begin{array}{c}\Delta T_{\mathrm{s}} \\
\boldsymbol{\theta}_{*}\end{array}$ & $\begin{array}{l}d_{u} \\
H\end{array}$ & $\begin{array}{l}d_{i} \\
H\end{array}$ & $\begin{array}{l}d_{T} \\
H\end{array}$ & $\begin{array}{c}U D \\
v_{*} H\end{array}$ & $\begin{array}{c}T D \\
\theta_{*} H\end{array}$ & $\begin{array}{c}U T D \\
v_{*} \theta_{*} H\end{array}$ & $\begin{array}{c}U U D \\
v_{*}^{2} H\end{array}$ \\
\hline B02 & 0.503 & 4.51 & $20 \cdot 43$ & 11 & $9 \cdot 68$ & 7 & $27 \cdot 7$ & $7 \cdot 88$ & 71 & $124 \cdot 1$ \\
\hline & 0.011 & & $0 \cdot 17$ & 0 & 0.52 & 0 & 0.25 & 0.38 & $2 \cdot 6$ & 7.2 \\
\hline B04 & 0.43 & $3 \cdot 422$ & $18 \cdot 51$ & $7 \cdot 333$ & $6 \cdot 5$ & $4 \cdot 03$ & 13.97 & $2 \cdot 93$ & 20.53 & $43 \cdot 2$ \\
\hline & $0 \cdot 007$ & 0.082 & $0 \cdot 15$ & 0 & 0.62 & $0 \cdot 14$ & 0.062 & 0.22 & 0.94 & $2 \cdot 6$ \\
\hline B07 & $0 \cdot 397$ & 2.90 & $17 \cdot 39$ & $5 \cdot 33$ & $4 \cdot 75$ & $3 \cdot 03$ & 7.998 & 1.574 & $9 \cdot 4$ & 20 \\
\hline \pm & 0.007 & 0.11 & $0 \cdot 1$ & 0.24 & 0.72 & $0 \cdot 13$ & 0. & & & 1.8 \\
\hline B10 & 0.371 & $2 \cdot 5$ & 16.7 & $4 \cdot 1$ & 3.91 & $2 \cdot 1$ & & $0 \cdot 858$ & 4. & $12 \cdot 36$ \\
\hline \pm & 0.01 & 0.093 & $0 \cdot 14$ & 0.2 & $1 \cdot 1$ & 0.17 & $0 \cdot 014$ & 0.079 & 0.6 & 0.95 \\
\hline $310^{*}$ & $0 \cdot 375$ & $2 \cdot 55$ & $16 \cdot 84$ & 4.48 & 4.03 & 2.47 & $4 \cdot 76$ & $1 \cdot 17$ & $5 \cdot 63$ & $12 \cdot 4$ \\
\hline \pm & 0.025 & 0.29 & $0 \cdot 35$ & $1 \cdot 2$ & 0.77 & 0.63 & $1 \cdot 7$ & $2 \cdot 3$ & 2 & $3 \cdot 5$ \\
\hline B20 & $0 \cdot 344$ & $2 \cdot 0 t$ & $16 \cdot 1$ & $2 \cdot 93$ & $2 \cdot 62$ & & & & & 4.71 \\
\hline & $0.0 \mathrm{r}$ & & 0 & 0.26 & 0.49 & & & & & 0.27 \\
\hline B30 & 0.34 & 1.9 & $16 \cdot 11$ & $2 \cdot 16$ & $1 \cdot 88$ & $0 \cdot 9$ & & 0.2 & & $2 \cdot 95$ \\
\hline \pm & 0.007 & 0.063 & $0 \cdot 17$ & 0.13 & 0.22 & 0.065 & $0 \cdot 0$ & 0 . & 0. & $0 \cdot 13$ \\
\hline B45 & 0.396 & $2 \cdot 26$ & $16 \cdot 18$ & 1.56 & 1.25 & $0 \cdot 5$ & $1 \cdot 3$ & 0.229 & & $2 \cdot 34$ \\
\hline \pm & $0 \cdot 015$ & $0 \cdot 13$ & $0 \cdot 2$ & 0.27 & $0 \cdot 12$ & $0 \cdot($ & & & & 0.21 \\
\hline B90 & 0.552 & $3 \cdot 28$ & $17 \cdot 54$ & 0.773 & 0.692 & $0 \cdot 3$ & & & & $2 \cdot 51$ \\
\hline \pm & 0.016 & 0.11 & 0.27 & 0.038 & 0.055 & 0 & 0.011 & 0.022 & $0 \cdot 2$ & $0 \cdot 14$ \\
\hline R02 & 0.444 & $4 \cdot 42$ & $24 \cdot 19$ & 11 & $9 \cdot 61$ & $6 \cdot 8$ & 27.67 & $6 \cdot 6$ & 66 & 119.9 \\
\hline & $0 \cdot 0$ & & & 0 & $0 \cdot 6$ & 0 & & & & $7 \cdot 6$ \\
\hline R 10 & $0 \cdot 334$ & $2 \cdot 6$ & $21 \cdot 11$ & $4 \cdot 17$ & $4 \cdot 0$ & 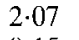 & & 0.736 & $4 \cdot$ & 11.91 \\
\hline \pm & 0.004 & 0.05 & $0 \cdot 1$ & 0. & $0 \cdot 8$ & & & & & 0.55 \\
\hline R30 & 0.32 & $2 \cdot 24$ & $20 \cdot 7$ & $2 \cdot($ & 1.99 & & & & & $3 \cdot 3$ \\
\hline \pm & $0 \cdot 007$ & 0.08 & & $0 \cdot 2$ & 0.69 & & & 0.009 & & $0 \cdot 15$ \\
\hline R45 & 0.361 & $2 \cdot 5$ & $20 \cdot 82$ & & $1 \cdot 178$ & & & 0.18 & & $2 \cdot 62$ \\
\hline \pm & 0.01 & 0.099 & $0 \cdot 14$ & $0 \cdot 12$ & $0 \cdot 084$ & 0.057 & 0.002 & $0 \cdot 01$ & 0. & $0 \cdot 18$ \\
\hline R90 & 0.484 & $3 \cdot 667$ & $22 \cdot 64$ & 0.74 & 0.711 & $0 \cdot 3$ & 0.9 & 0.238 & & $2 \cdot 7$ \\
\hline \pm & $0 \cdot 011$ & 0.093 & 0.32 & 0.064 & 0.077 & 0 & $0 \cdot 012$ & 0.025 & 0.22 & $0 \cdot 18$ \\
\hline $\mathrm{C} 10$ & 0.568 & & & & & & & & & $10 \cdot$ \\
\hline \pm & 0.0 & & 0.0 & 0 & 0.4 & & & & & 0.48 \\
\hline E10 & 0.266 & $2 \cdot 785$ & $34 \cdot 44$ & 4 & $3 \cdot 34$ & 1.84 & & & 3.87 & $12 \cdot 3$ \\
\hline \pm & 0.007 & $0 \cdot 11$ & & 0 & 0.33 & $0 \cdot 19$ & & & & 0.82 \\
\hline F10 & $0 \cdot 203$ & 2.938 & $63 \cdot 03$ & $3 \cdot 7$ & 2.95 & $1 \cdot 6$ & $5 \cdot 6$ & & $3 \cdot 41$ & 12.98 \\
\hline \pm & 0.003 & 0.066 & 0.42 & $0 \cdot 17$ & $0 \cdot 19$ & 0 & 0 & $0 \cdot 014$ & 0.22 & 0.38 \\
\hline D10 & & & & & & & & & & 11.94 \\
\hline & & & & & & & & & $0 \cdot 36$ & $0 \cdot 31$ \\
\hline D90 & 0.621 & 3.95 & $16 \cdot 88$ & 0.767 & 0.722 & 0.277 & 0.967 & 0.375 & 2 & $2 \cdot 48$ \\
\hline \pm & 0.024 & 0.24 & 0.41 & 0.061 & $0 \cdot 04$ & 0.018 & 0.022 & 0.026 & $0 \cdot 24$ & 0.21 \\
\hline
\end{tabular}

Case B10* is the same as B10 but without control of steady state. For each case, the first line contains the mean values and the second line the corresponding standard deviations.

already much smaller than the value $\Delta T_{s}$ at the wall itself (see Table 2). This indicates much larger diffusivities inside the boundary layer than close to the wall. For the same reason, the velocity maximum is reached very close to the wall. The height $d_{T}$ of the first zero of the temperature profile is larger than half the height $d_{u}$ of the first zero of the velocity profile. In absolute terms, the maximum velocity, the temperature deviation near the surface and the depth of the UBL closely agree with observations as mentioned in the introduction, if we use the estimates of the scales $v_{*}, \theta_{*}$ and $H$ as explained after Eq. (1). Both the velocity and the temperature profiles show negative values at the outer edge of the boundary layer. As for the laminar flow, negative temperature deviations are caused by up-slope advection of cold fluid. The up-slope motion is driven counter to the buoyancy forces by upward momentum transport from the region with maximum velocity. The cold fluid in turn retards the motion and causes negative velocity further outside where the momentum transfer no longer overcomes the retarding force of 


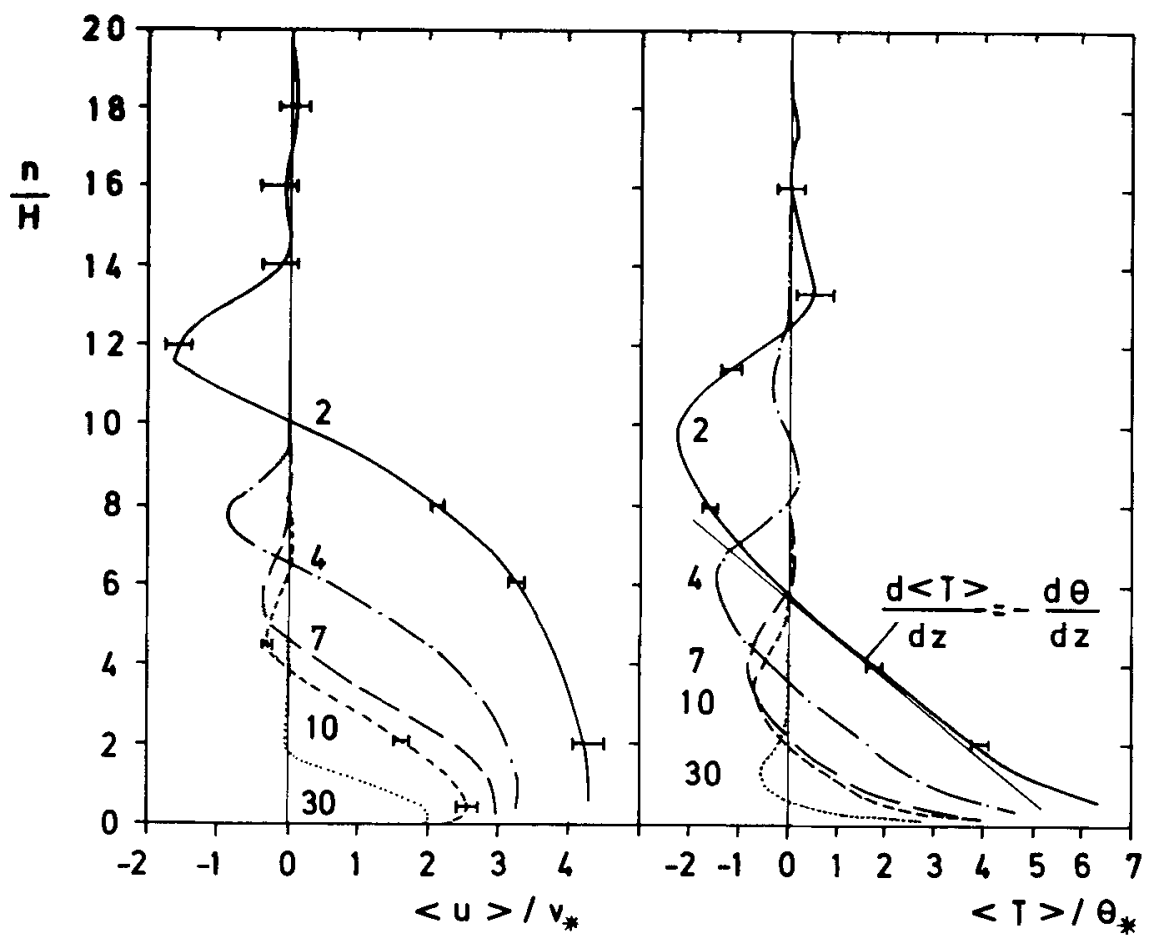

Figure 3. Mean velocity and mean temperature versus normal coordinate $n$ for moderately large slope-angles $\alpha$ shown in degrees on the curves. The error bars at some curves signify the standard deviations. The thin line in the right panel corresponds to the well-mixed situation.

buoyancy. Thus the general trend is as expected from the laminar solution depicted in Fig. 1 but the different form of the profiles requires further explanation.

As indicated in Fig. 3, the temperature profile gradient is close to $\cos \alpha d \theta / d z$ for $\alpha \leqslant 10^{\circ}$ over a substantial fraction of the boundary layer up to $n=d_{T}$. Such a gradient is to be expected for the temperature deviation $T$ when the UBL contains a layer which is convectively well mixed. Such a well-mixed layer can be seen for $\alpha \leqslant 10^{\circ}$ but not for larger angles. The rather flat velocity profile for $\alpha=2^{\circ}$ in the range $1 \leqslant n / H \leqslant 6$ appears to be a further consequence of the mixing. Also the observations of Deardorff and Willis (1987) and Zhou et al. (1985) exhibit a mixed layer. The mixed layer is topped by an inversion. At such inversions turbulent fluxes get small, relative to the turbulent transports inside the mixed layer. The effective diffusivity might even get negative, as we will see to be the case for small angles. This enables the flow to form strong local minima in the velocity and temperature profiles at the outer edge of the mixed layer. Also Deardorff and Willis (1987) and Zhou et al. (1985) observe strong return flows, but it is not clear how far these are affected by baroclinicity above the mixed layer. A mixed layer does not form and related inversion effects are weaker at larger angles. This explains that there are differences in the form of the profiles for various angles.

The effective boundary-layer thickness as measured by $d_{u}$ gets strongly reduced for steeper slopes and this is in qualitative agreement with Prandtl's laminar solution. Therefore, plots of the profiles for large angles require a different scaling as in Fig. 4. (The curves do not cross exactly in one point because the mean of $d_{u}$, which is used for normalization, differs slightly from the height of the zero of the mean velocity profile.) We observe from this plot that the profiles for $\alpha>10^{\circ}$ are quite similar in these scales except for a steepening of profiles near the wall for very large slope-angles. The return flow gets small while the range with negative temperature deviations gets rather large 


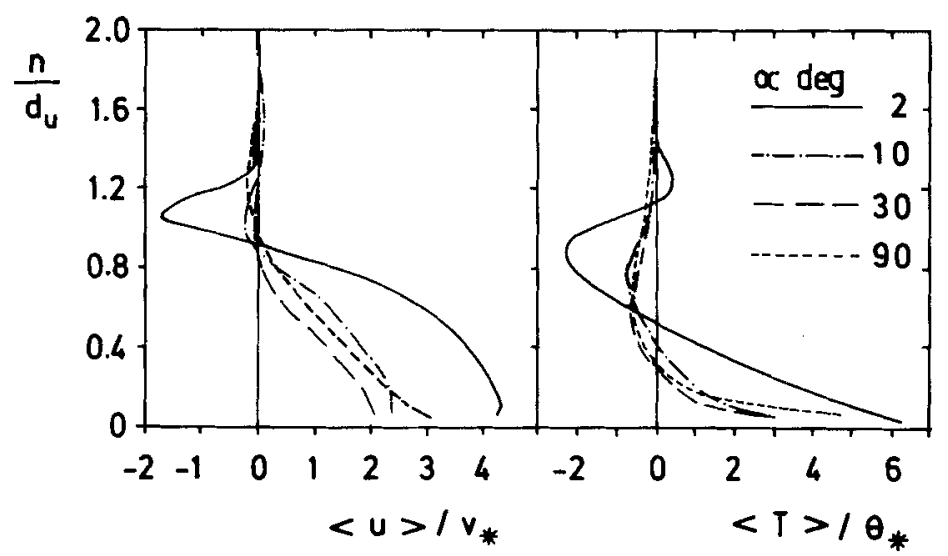

Figure 4. Mean velocity and mean temperature versus normal coordinate $n$ normalized with $d_{u}$, the mean position of the first zero value of the mean velocity, for small and large slope-angles $\alpha$.

for $\alpha>10^{\circ}$. For such large angles the profile shape is closer to the laminar solution than for small angles.

\section{(c) Turbulence statistics}

The turbulence covariances reach their final magnitude rather quickly. The timescale of the approach to equilibrium corresponds to the turn-over time $d_{u} /\langle E\rangle^{1 / 2}$, where $E=u_{i}^{\prime 2} / 2$ is the turbulence energy. However, the shape of the mean profiles oscillates for much longer times owing to weakly damped gravity waves outside the boundary layer. Figure 5 depicts various turbulence statistics versus the normal coordinate for $\alpha=$ $10^{\circ}$ as obtained from the fine grid, case D10. The error bars signify reasonably small uncertainty due to remaining variations around steady state. Most profiles include both the contributions from the resolved motion scales and the SGS motions. Only the pressure diffusion terms are without SGS contributions. The dashed curves show that the contributions from the SGS model are small, except near the surface where SGS motions dominate. The results can best be understood from Fig. 6, which shows various contributions to the balance of turbulent kinetic energy. Some of the terms in this balance are obtained by finite differences of mean profiles and, therefore, are reliable only up to errors of about $\pm 0 \cdot 1 \mathrm{H} / v_{*}^{3}$.

As can be seen from Reynolds stress transport equations (e.g. Launder 1975), the variances of up-slope velocity fluctuations are excited mainly by the shear production $S$, while the normal velocity fluctuations $\left\langle w^{\prime 2}\right\rangle$ are directly produced solely by the buoyancy flux $\beta g\left\langle w^{\prime} T^{\prime}\right\rangle \cos \alpha$. The third velocity component $v^{\prime}$ obtains its energy by means of pressure-strain correlations. The momentum flux $\left\langle u^{\prime} w^{\prime}\right\rangle$ is negative at the surface, i.e. turbulence transports momentum from the interior to the wall. However, the flux drops quickly to zero, and reaches an even larger but positive value at the negative slope of the velocity profile. Note that the divergence of the stress balances the buoyancy force in the up-slope direction, see Eq. (3). Hence the flux $\left\langle u^{\prime} w^{\prime}\right\rangle$ reaches its maximum value at $n=d_{T}$, i.e. at the height of zero temperature deviation. The correlation coefficient $\left\langle u^{\prime} w^{\prime}\right\rangle /\left(\left\langle u^{\prime 2}\right\rangle\left\langle w^{\prime 2}\right\rangle\right)^{1 / 2}$ reaches the rather large value of 0.36 which is indicative of large shear contributions to turbulence as also noted by Deardorff and Willis (1987). The product of flux and negative shear provides the shear generation of turbulence as plotted in Fig. 6. It is large near the wall and near $n / H \cong 2.5$ but drops to zero at $n \cong 0.4 H$ and $n \cong 7 \mathrm{H}$. The shear production directly excites horizontal velocity variance $\left\langle u^{\prime 2}\right\rangle$ which, therefore, exhibits maximum values near the wall and near $n \cong 2.5 \mathrm{H}$. The rather large values of $\left\langle v^{\prime 2}\right\rangle$ near the surface indicate an efficient transfer of energy by pressure-strain 

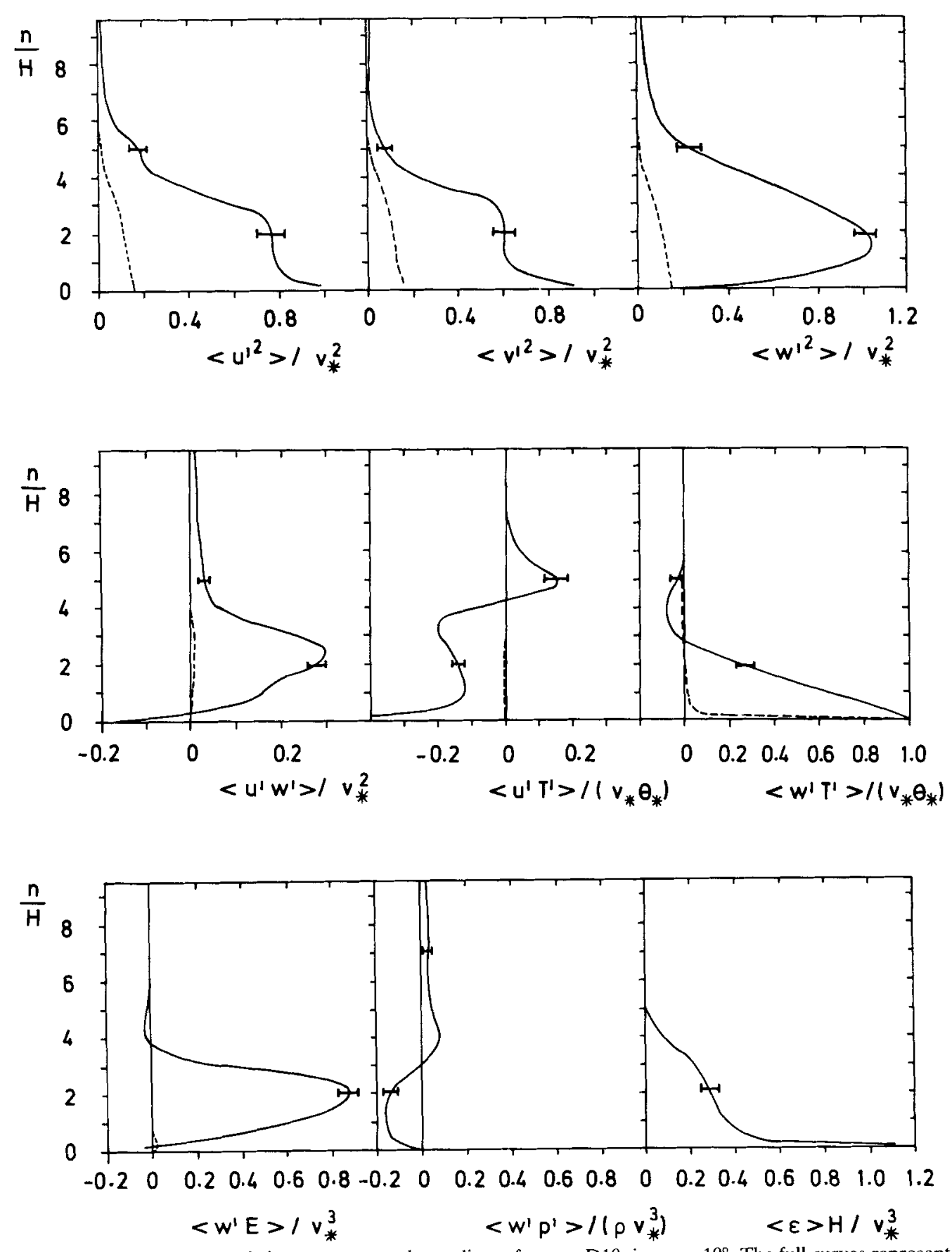

Figure 5. Turbulence statistics versus normal coordinate for case D10, i.e. $\alpha=10^{\circ}$. The full curves represent the sum of resolved and SGS (subgrid-scale) contributions at steady state. The error bars indicate the standard deviation due to remaining fluctuations relative to steady state. The dashed curves depict SGS contributions. 


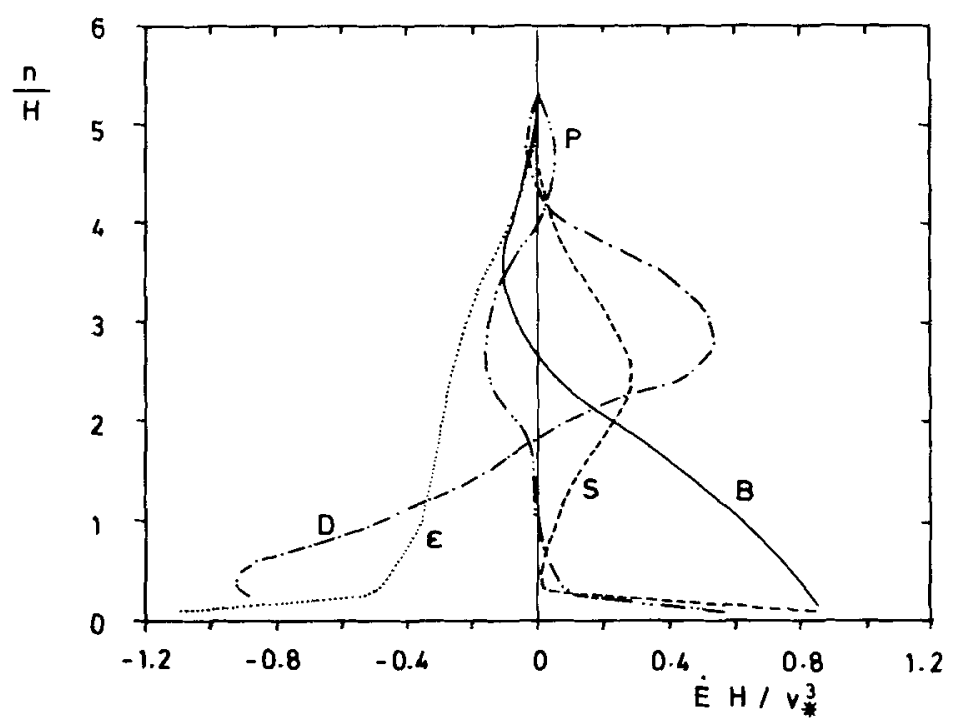

Figure 6. Contributions to the turbulent kinetic energy budget versus normal coordinate for case D10. B: $\beta g\left(\left\langle w^{\prime} T^{\prime}\right\rangle \cos \alpha+\left\langle u^{\prime} T^{\prime}\right\rangle \sin \alpha\right)$, buoyancy production; S: $-\left\langle u^{\prime} w^{\prime}\right\rangle d\langle u\rangle / d n$, shear production; $\varepsilon:-\left\langle c_{m m} \frac{D}{E^{\prime \prime}} 3 / 2\right\rangle$ $\ell\rangle$, dissipation rate; $\mathrm{D}:-d\left\langle w^{\prime} E^{\prime}\right\rangle / d n$, turbulent diffusion; $\mathrm{P}:-\rho^{-1} d\left\langle w^{\prime} p^{\prime}\right\rangle / d n$, wavy transport.

correlations. This effect is presumably enhanced by buoyancy effects, because eddies with large values of $u^{\prime}$ tend to be rather cool (see the correlation $\left\langle u^{\prime} T^{\prime}\right\rangle$ ) and thus are more easily deflected in the $y$-direction than in the normal direction.

The momentum flux $\left\langle u^{\prime} w^{\prime}\right\rangle$ apparently follows the gradient of the mean velocity profile near the surface, but does not drop to zero at the height $n \cong 4 \cdot 5 H$ of the velocity minimum (see Fig. 3). Thus we observe strong departure from gradient transports and the negative ratio of the momentum flux relative to the velocity shear would imply negative eddy viscosity in this region. It appears as if the momentum transport is driven by the mean velocity difference between the mixed layer and the outer domain and not so much by local gradients. Hence, the effective viscosity does not smooth out, but enhances the strong backward flow at the outer edge of the boundary layer. The normal heat flux takes its prescribed value (unity in normalized terms) at the surface and then decays about linearly to zero. The approximately linear decrease reflects a uniform heating rate across this layer which is balanced by a close to uniform up-slope advection of cold fluid. The heat flux then gets negative and reaches a minimum value of about $-0 \cdot 1 Q_{\text {s }}$ at a height $d_{i} \cong 3 \cdot 8 \mathrm{H}$. This height corresponds to the usual height definition of a horizontal convective boundary layer and the negative heat flux is caused by entrainment as explained, e.g., in Schmidt and Schumann (1989). This entrainment heat flux reduces vertical velocity fluctuations near this height but this negative contribution to the energy balance is outweighed by other terms, (see Fig. 6). The structure of the up-slope heat flux is most complicated. It can hardly be explained in terms of gradient models which would predict a small negative value for this flux. Therefore, we refer to the transport equation for this term,

$$
\frac{\partial\left\langle u^{\prime} T^{\prime}\right\rangle}{\partial t}=P+\Phi
$$

in which $P$ denotes the production terms

$$
P=-\left\langle u^{\prime} w^{\prime}\right\rangle \frac{d\langle T+\theta\rangle}{d n}-\left\langle w^{\prime} T^{\prime}\right\rangle \frac{d\langle u\rangle}{d n}-\left\langle u^{\prime 2}\right\rangle \frac{d \theta}{d z} \sin \alpha+\beta g\left\langle T^{\prime 2}\right\rangle \sin \alpha
$$


and $\Phi$ comprises diffusion terms plus pressure-temperature and molecular destruction terms. For small diffusion, models usually predict $\Phi=-\left\langle u^{\prime} T^{\prime}\right\rangle / \tau$ where $\tau$ is a suitable time scale of turbulence. Thus, in steady state and for small diffusion terms, such models predict that the up-slope heat flux is proportional to $P$.

In $P$, the last term due to buoyancy always causes positive trends, while the third term is always negative but both have large magnitude only for steep slopes. Near the surface, the first two production terms have different signs and it appears that the first term is responsible for the negative up-slope heat flux in that region. The two first production terms change sign at a certain distance from the wall. At the outer edge of the boundary layer, the first two production terms both become positive and this explains qualitatively the trends which we observe for this flux in Fig. 5. The up-slope turbulent heat flux contributes to buoyancy production of kinetic energy as $\beta g \sin \alpha\left\langle u^{\prime} T^{\prime}\right\rangle$. This term is rather small because of the small angle but notable in that it causes the normalized buoyancy term $B$ to be smaller than unity at the wall, (see Fig. 6). Most authors have neglected this effect, which can be large for large angles, both in turbulence models (Rao and Snodgrass 1981; Brehm 1986) and evaluations of laboratory measurements (Deardorff and Willis 1987).

Figure 5 also shows large turbulent transports of kinetic energy of the diffusive type, $\left\langle w^{\prime} E\right\rangle$. The pressure transport $\left\langle w^{\prime} p^{\prime} / \rho\right\rangle$ of kinetic energy is small inside the boundary layer and of opposite sign but becomes important at the outer edge of the boundary layer. It is carried by gravity waves which radiate energy far away from the UBL and it is this effect which requires proper boundary conditions in the simulations at the outer boundary to avoid reflections. Obviously, the boundary condition used in this simulation provides a good approximation in this sense.

Deardorff and Willis (1987) assumed that $\left\langle w^{\prime} p^{\prime} \mid \rho\right\rangle \cong-0 \cdot 4\left\langle w^{\prime} E\right\rangle$. A factor of $0 \cdot 2$ instead of 0.4 would be better supported by our results. The estimate of the dissipation rate given by these authors considerably exceeds our LES results shown in Figs. 5 and 6, in particular near the outer edge of the UBL. Deardorff and Willis (1987) also find a much stronger shear production rate in the outer part of the boundary layer than we compute (Fig. 6) and show that about $10 \%$ of this shear production is due to non-zero values of the mean normal velocity in the experiment. Also, the measured velocity variances are larger than our computed results by about a factor of two. Deardorff and Willis (1987) mention 'very important' advection terms but do not quantify the possibly large advective contributions in the laboratory case which will arise because of the decreasing height of the boundary layer in the up-slope direction in the experiment. These advection effects, shear production due to sinking motion, and the overestimate of the pressure-diffusion energy-transport explain the differences between the experimental and numerical results.

Zhou et al. (1985) also show a plot of the turbulence kinetic energy budget which is similar to our Fig. 6. For example, both the LES and the observations show that dissipation has a rather flat profile and that diffusional loss of energy exceeds dissipation in the lower third of the layer. The LES results are closer to these measurements than to those of Deardorff and Willis.

Figure 7 depicts the same turbulence quantities as obtained from the coarse-grid simulations Bxx for a sequence of angles. To make the results comparable in spite of the strong variation of the boundary-layer thickness, we have normalized the normal coordinate by the thickness $d_{u}$ of the velocity layer. For clarity, this figure contains the SGS contributions only in one panel. Further SGS results are plotted separately in Fig. 8 . The relative fraction of the SGS contributions are larger for the coarse grid than in Fig. 5. On average over the whole layer, the SGS energy amounts to about $21 \%$ of the total 

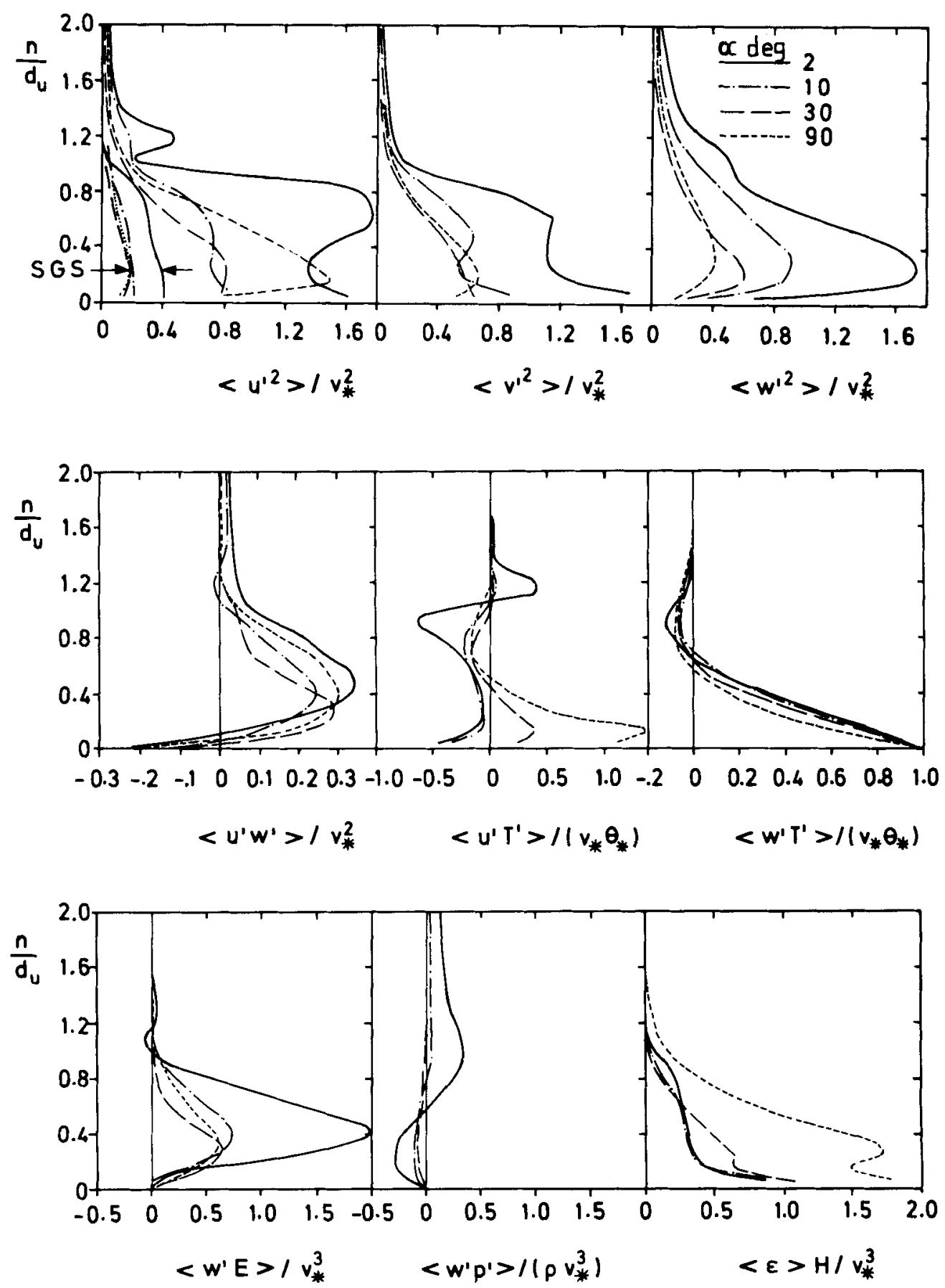

Figure 7. Turbulence statistics versus normalized normal coordinate for cases B02, B10, B30 and B90 with varying slope-angles $\alpha$. For $\left\langle u^{\prime 2}\right\rangle$, the SGS contributions are given. 


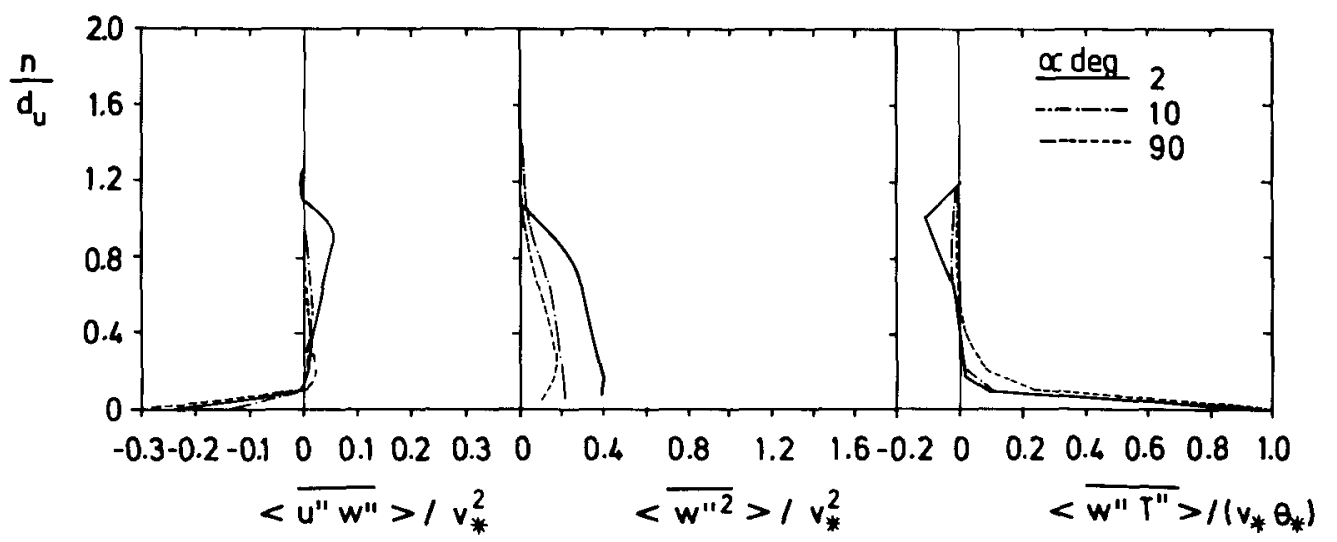

Figure 8. SGS contributions to selected turbulence covariances versus normal coordinate $n$ for cases B02, $\mathrm{B} 10$ and $\mathrm{B} 90$.

turbulence energy in all coarse-grid cases Bxx but it is $14 \%$ for case D10 and $18 \%$ for case D90. A variation according to $\Delta^{2 / 3}$ was to be expected. Even for the coarse grids, the SGS contributions do not dominate the results, except for the momentum transport at the surface. For $\alpha=10^{\circ}$, the results of the coarse grid shown in Fig. 7 are close to those obtained from the fine grid (see Fig. 5) which further supports the validity of the results. From Fig. 7 we find strong qualitative variations in the turbulence profiles with angle. The up-slope variance first decreases and then increases with increasing angle. For $\alpha=2^{\circ}$, strong secondary maxima are found above and below the velocity minimum. These secondary maxima are absent at large slope-angles. The normal velocity variance decreases more or less monotonically with the angle. The momentum flux profiles show smaller changes. The up-slope heat flux near the surface changes sign with increasing angle. This means that the product of heat flux times the velocity shear represents the dominating generation term for small angles, while the buoyancy term generates most of this flux for steep slopes. At small angles the up-slope heat flux diminishes the turbulence production whereas at large slope-angles it becomes the dominant contribution to positive buoyancy generation of turbulence. Note that $\left\langle u^{\prime} T^{\prime}\right\rangle$ for $\alpha=90^{\circ}$ gets even larger than $Q_{\mathrm{s}}$, and that this heat flux enhances the production of $\left\langle u^{\prime 2}\right\rangle$. The normal heat flux profile is close to linear near the wall at small angles corresponding to flat velocity profiles whereas the heat flux deviates more strongly from a linear decrease at large angles where the velocity profile gets steeper. The turbulent transport of kinetic energy is large for small angles but less important for large angles, i.e. the turbulence is more in local equilibrium at large angles than at small angles. The local dissipation rate increases with increasing angle and thus counterbalances stronger production of energy (by shear and up-slope heat flux) at large angles.

The integral contribution from shear to the energy balance amounts to about $40 \%$ of the whole forcing for all angles from $2^{\circ}$ to $20^{\circ}$, and then gradually increases to reach about $70 \%$ for $\alpha=90^{\circ}$. Thus, shear is important at all angles while buoyancy forcing dominates up to about $20^{\circ}$ but is still important for $90^{\circ}$.

In Fig. 9, extreme values of the turbulence covariances are plotted, as normalized by the convective velocity scale $w_{*}=\left(\beta g Q_{s} d_{i}\right)^{1 / 3}$. In this normalization the variances should stay constant if only buoyancy generates energy. It should also stay constant if shear contributes a fixed fraction to turbulence production, which is the case for moderate slope-angles. The results show that the normalized variance extrema are fairly constant with angle for $\alpha \leqslant 20^{\circ}$ but then the variances, in particular $\left\langle u^{\prime 2}\right\rangle$, increase strongly owing to the growing contribution of shear to turbulence at large angles. For $\alpha \leqslant 20^{\circ}$, the 


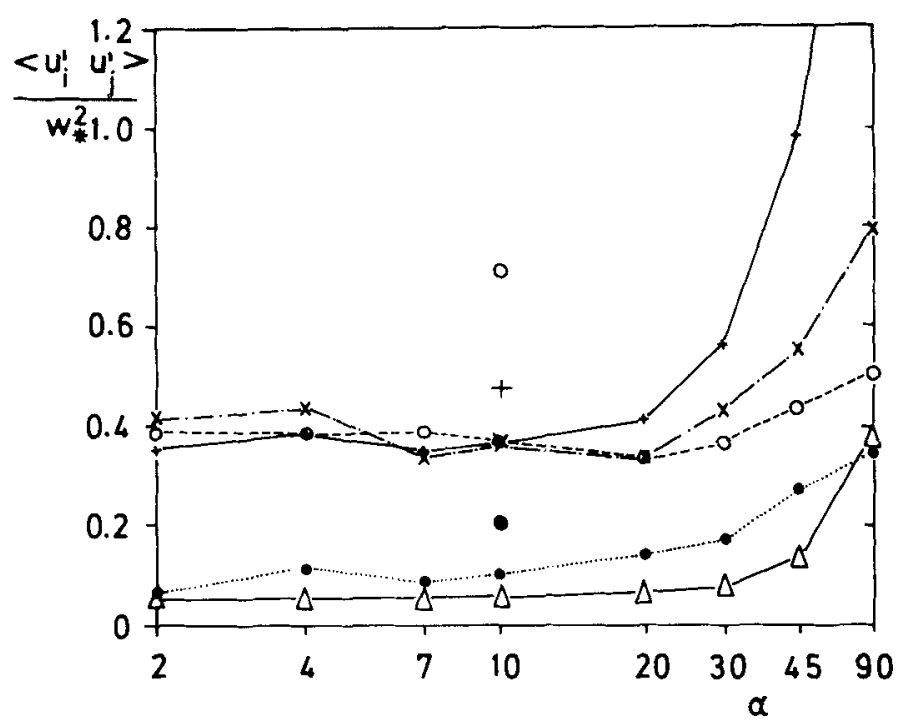

Figure 9. Maximum values of the profiles of turbulence covariances normalized by the convective velocity $w_{*}=\left(\beta g Q_{s} d_{i}\right)^{1 / 3}$ versus the logarithm of $\sin \alpha$. Full curve with $+:\left\langle u^{\prime 2}\right\rangle$; dash-dotted curve with $x:\left\langle v^{\prime 2}\right\rangle$; dashed curve with $0:\left\langle w^{\prime 2}\right\rangle$; dotted curve with $\bullet:\left\langle u^{\prime} w^{\prime}\right\rangle$. The full curve with $\Delta$ shows the negative momentum flux at the surface, i.e. $u_{*}^{2}$. The three isolated symbols for $\alpha=10^{\circ}$ depict the measurements of Deardorff and Willis (1987) from experiments with variable layer thickness. The maximum value of $\left\langle u^{\prime 2}\right\rangle / w_{*}^{2}$ for $\alpha=90^{\circ}$ (not shown) amounts to $1 \cdot 85$.

variance maxima agree closely with results known from horizontal convective boundary layers, (see Schmidt and Schumann 1989). However, the profiles are different and the mean value of horizontal variances is smaller in the shearless horizontal case than in the UBL. For $\alpha=10^{\circ}$, the corresponding results of Deardorff and Willis (1987) are included in this figure. The results of the laboratory experiment are much larger than our computed results. In particular, the measurements show larger normal fluctuations than horizontal ones whereas the simulations predict the opposite. Again, this is presumably a consequence of up-slope advection and baroclinicity in the laboratory boundary layer. It is noteworthy, that the internal positive momentum flux $\left\langle u^{\prime} w^{\prime}\right\rangle$ is larger than the negative wall flux $-u_{*}^{2}$ which indicates that this type of flow will be only weakly sensitive to surface properties such as the surface roughness parameter $z_{0}$. The surface friction was not measured by Deardorff and Willis (1987). Both the experiment and the present simulations exhibit $\left\langle v^{\prime 2}\right\rangle$ to be nearly of the same magnitude as $\left\langle u^{\prime 2}\right\rangle$. Zhou et al. (1985) have measured the vertical velocity variance in the baroclinic convective boundary layer and find a maximum value $\left\langle w^{\prime 2}\right\rangle / w_{*}^{2}=0 \cdot 5$. This result and also the shape of the variance profile agree well with the LES results for small inclination angles.

\section{(d) Turbulent flow structure}

Figures 10 to 12 depict instantaneous flow fields for various field components, crosssections, angles and resolution. All results are taken from the final time of the simulations. The temperature plots in Fig. 10 depict the sum $T+\theta$ to simplify interpretation. All plots for comparable cross-sections use the same contour intervals. We have convinced ourselves that the results from case B10 are close to those from case $\mathrm{B} 10^{*}$, which was run without steady-state control, so that the resultant structures are not affected by the control scheme.

In Fig. 10, the temperature cross-sections clearly indicate the steepening of isotherms with increasing angle. The isotherms outside the turbulent layer are far from straight 


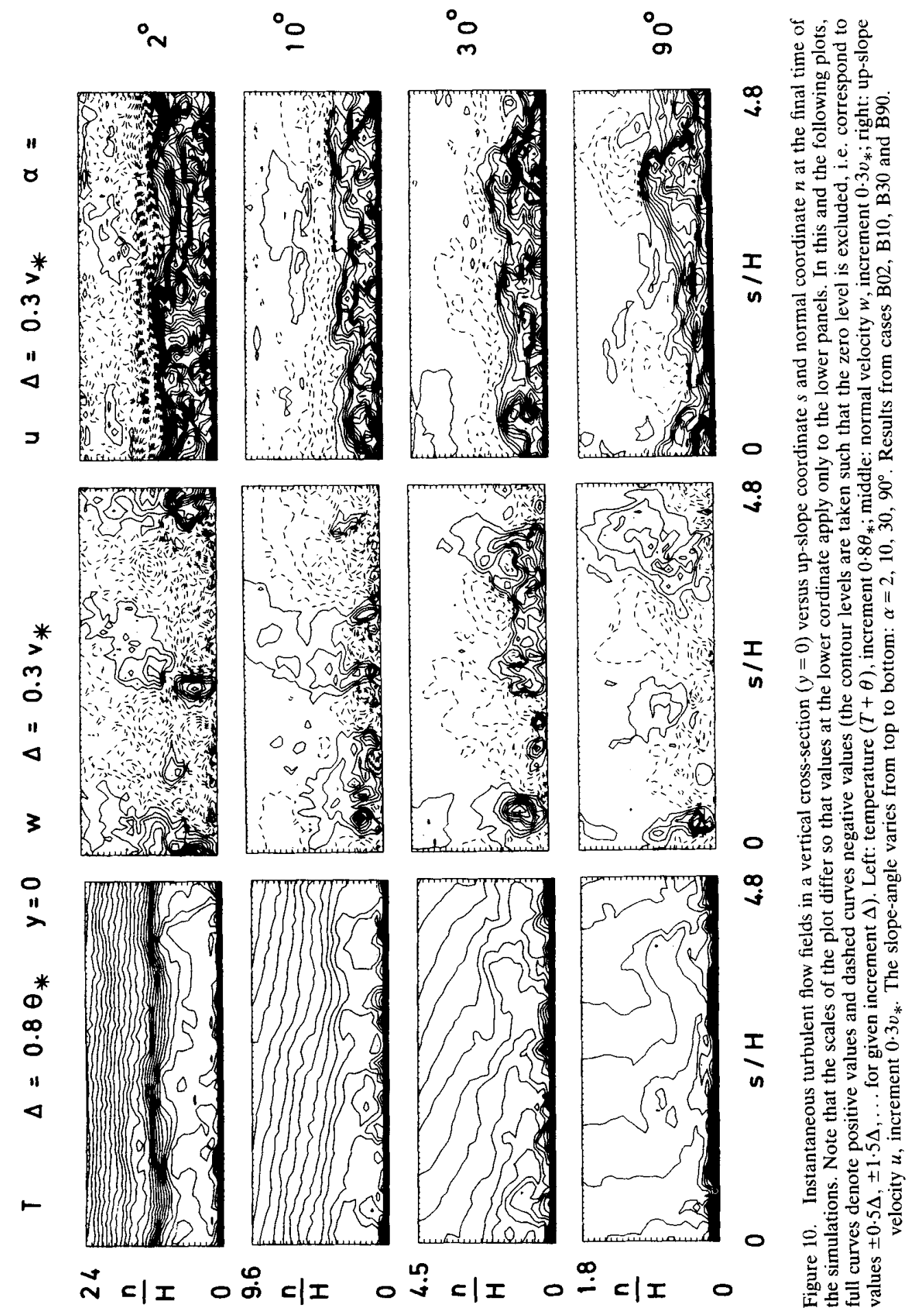


owing to wavy motions. For $\alpha=2^{\circ}$ and to lesser extent for $\alpha=10^{\circ}$, a wide layer of wellmixed temperature can be seen. The temperature increases strongly near the surface and the temperature variance is also largest in that layer. At the outer edge of the boundary layer the isotherms are narrowing, indicating the formation of a strong inversion, in particular for small angles. This structure is similar to what we know about the horizontal convective boundary layer. The normal velocity shows rather narrow up-draughts or ejections with large areas of slow motion towards the wall. This type of velocity skewness, which contributes to outward diffusion of kinetic energy, diminishes at larger angles. The up-slope velocity contours again reflect a well-mixed layer for small angles with a sharp reduction of $u$ at the inversion. With increasing angle, the up-slope motion and also the temperature contours indicate overturning waves as they are observed typically for strong Kelvin-Helmholtz instabilities. For $\alpha=90^{\circ}$, the horizontal wavelength approaches the size of the computational domain. Otherwise, the computational domain is large enough to cover all important scales of motion.

Figure 11 shows similar results for two angles from the fine-grid simulations D10 and D90. The structure is basically the same as shown by the coarse-grid simulations which is obviously important for the validity of the coarse-grid simulations. However, many more details can be identified. In particular, for $\alpha=10^{\circ}$, we find that the updraughts are not just uniform plumes but contain smaller scale bubbles of rising motion. This corresponds to results which have been identified by conditional sampling in Schmidt and Schumann (1989) over horizontal surfaces. For $\alpha=90^{\circ}$, Fig. 11 illustrates the very narrow shear layer at the surface which explains why this case is more sensitive to truncation errors than others. The Kelvin-Helmholtz waves, if present, appear to have a smaller wavelength in this simulation. The normal velocity shows motions which take their maximum further outside for $\alpha=90^{\circ}$ than for $\alpha=10^{\circ}$.

Figure 12 shows horizontal cross-sections of cases D10 and D90 at a distance $n / d_{u} \cong 0 \cdot 3$. In both cases this 'height' is below $d_{T}$. At this distance, the flow contains motions that are still rather small-scale, whereas larger-scale motions dominate further outside. The computational domain appears to be large enough to capture the most energetic small-scale motions. However. some large-scale coherence is notable. In particular, for $\alpha=10^{\circ}$, the large-scale components exhibit large spatial correlation in the cross-stream direction $y$. The up-slope distance between cross-slope rolls for $\alpha=10^{\circ}$ can be estimated from Fig. 12 to be about $10 \mathrm{H}$ or $2 \cdot 5 d_{i}$. It is thus interesting to note that Zhou et al. (1985) report a normalized peak wavelength for the vertical velocity of about $3 d_{i}$. A polygonal spoke pattern as found in the horizontal boundary layer by Mason (1989) and Schmidt and Schumann (1989) does not appear to be important for the present cases. For $\alpha=10^{\circ}$, the temperature fluctuations are obviously strongly correlated with normal velocity $w$ whereas for $\alpha=90^{\circ}$, the correlation with $u$ is much stronger. This difference is reflected in the magnitude of the normal or up-slope heat flux values, plotted in Fig. 7. At $\alpha=90^{\circ}$, large-scale coherence seems to be stronger in the up-slope direction. But roll vortices, if present, do not dominate the flow structure. Occasionally, a lambdatype structure can be observed with the corner pointing up-slope. However, such examples are not very frequent. Identification of travelling waves, as found by Gill and Davey (1969) and Clever and Busse (1977) from linear analysis, would require spacetime correlations which we have not evaluated.

Figures 13 and 14 contain autocorrelation functions

$$
R_{f f}\left(r_{1}, r_{2}, x_{3}\right)=\left\langle f\left(x_{1}, x_{2}, x_{3}\right) f\left(x_{1}+r_{1}, x_{2}+r_{2}, x_{3}\right)\right\rangle /\left\langle f^{2}\left(x_{1}, x_{2}, x_{3}\right)\right\rangle
$$

where the arguments of $f$ are evaluated modulo the domain size. The plots are constructed so that $\vec{r}=\left(r_{1}, r_{2}\right)=0$ corresponds to the centre of the panel, and show results for positive 


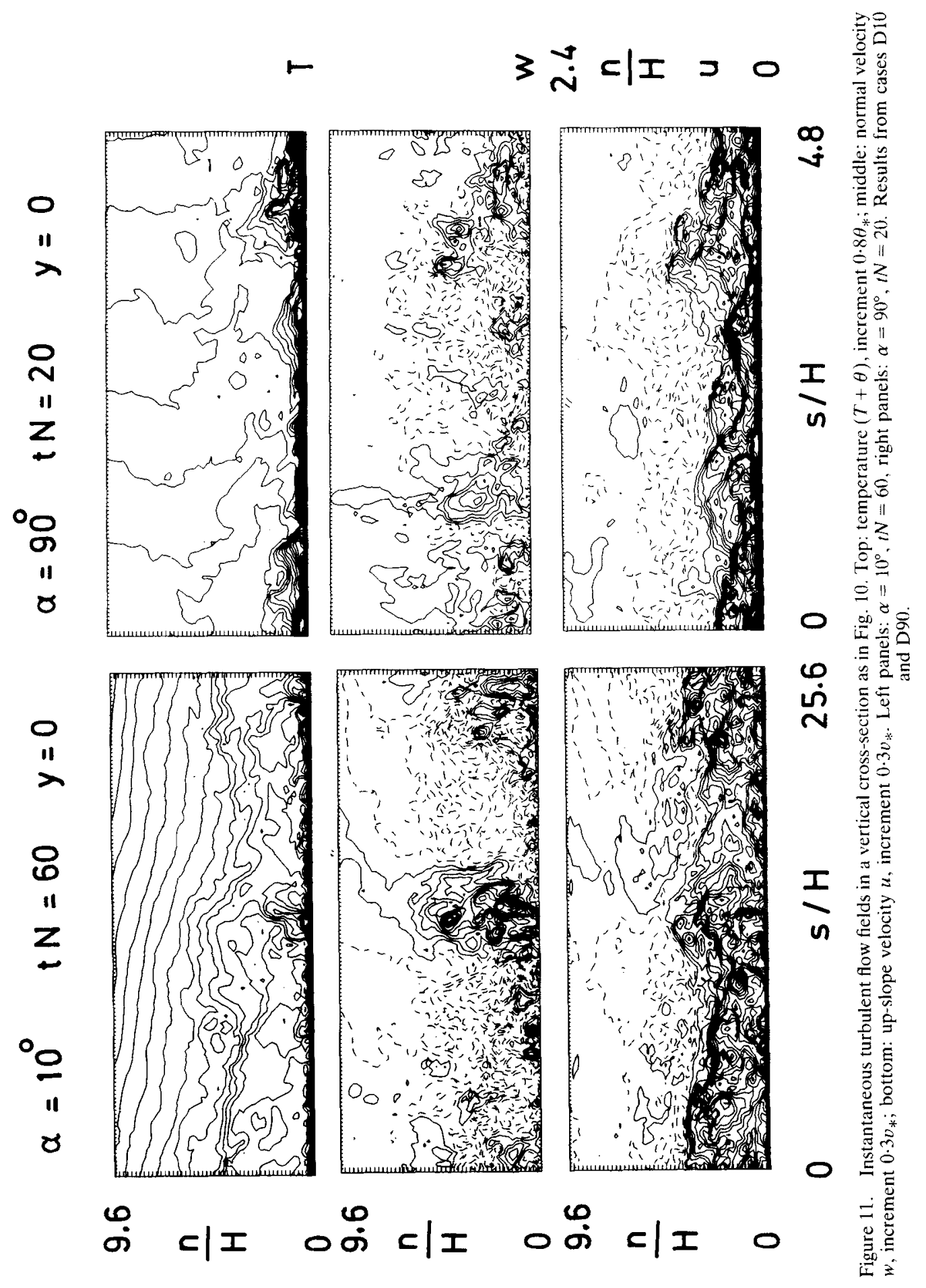




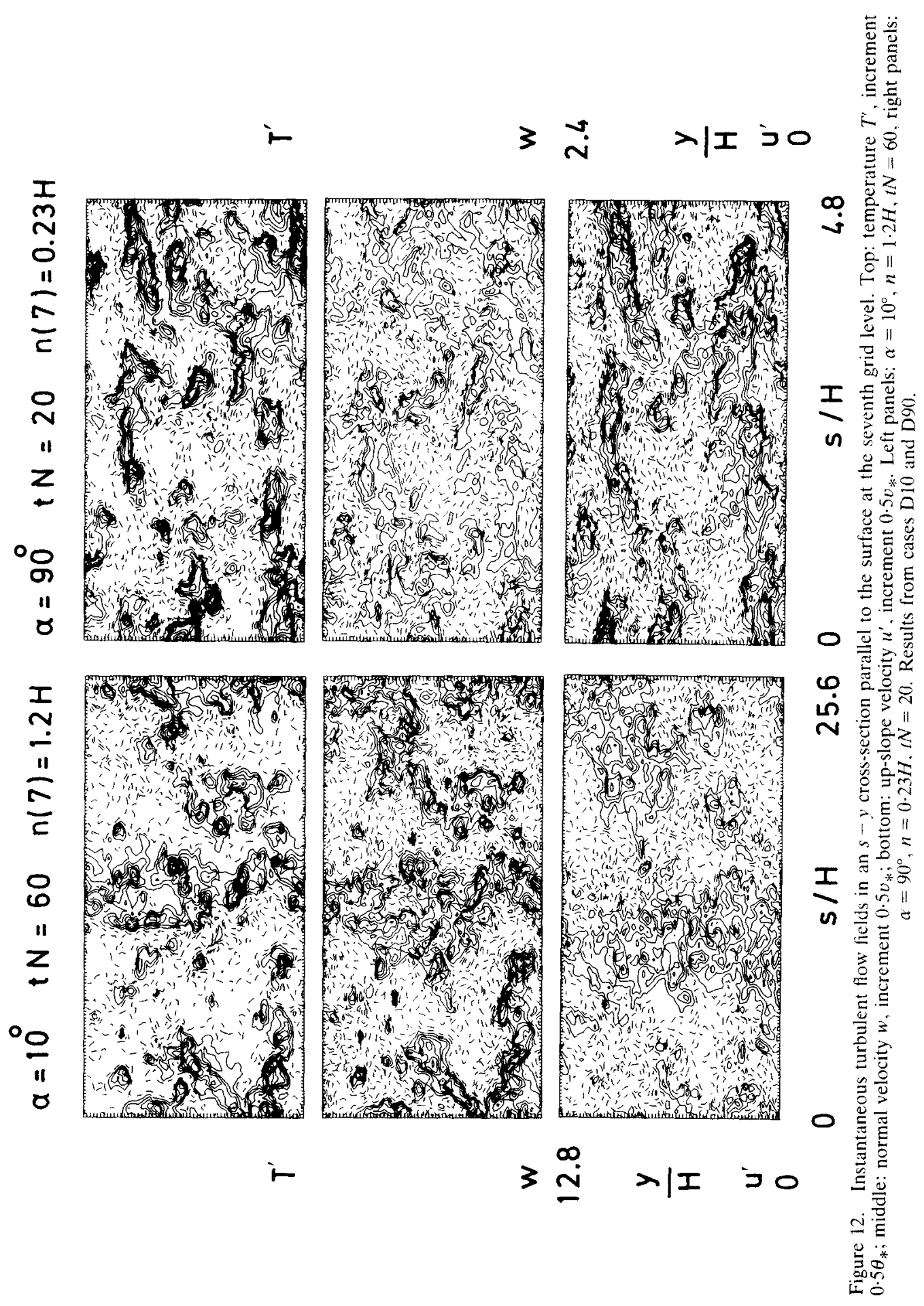




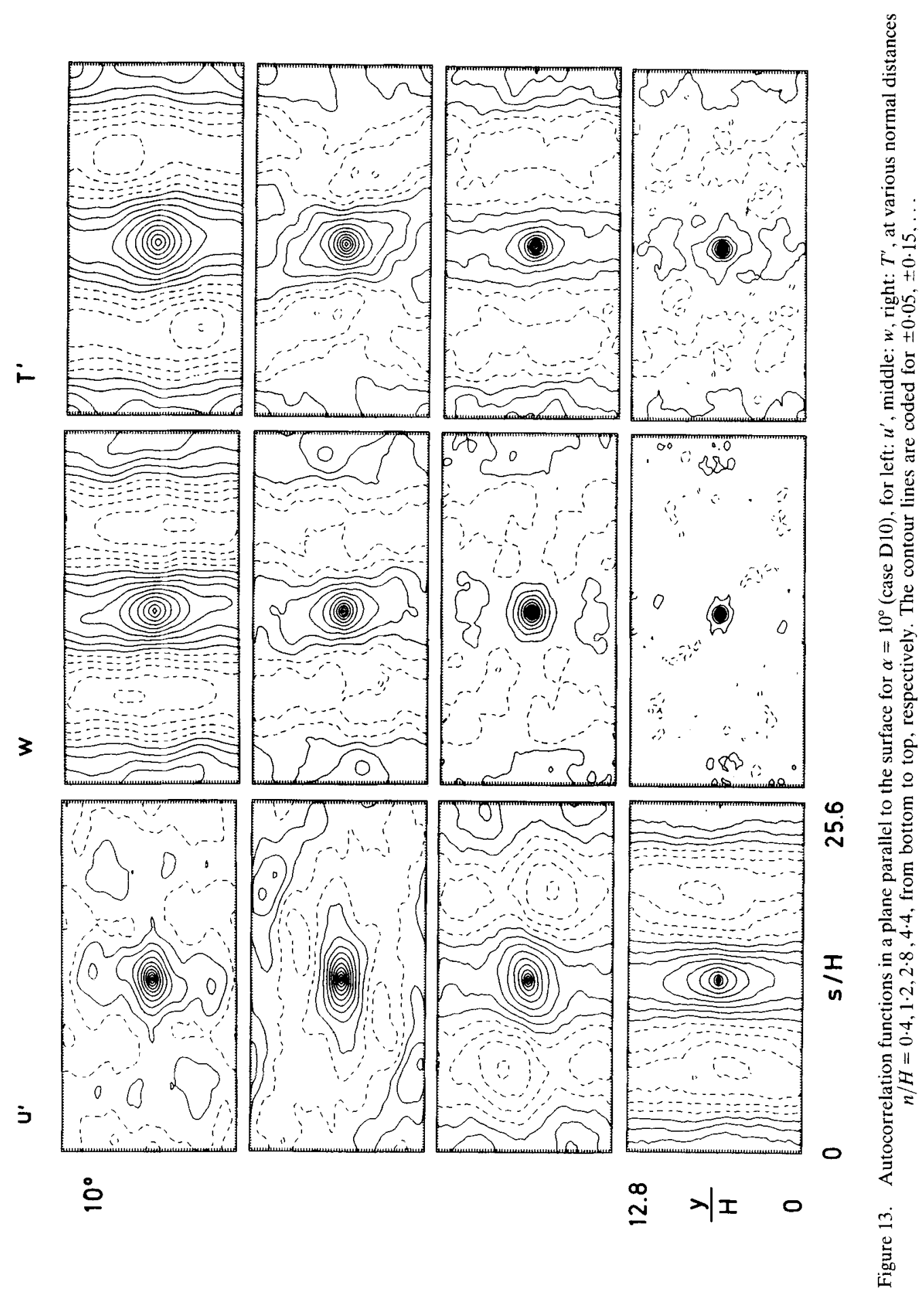




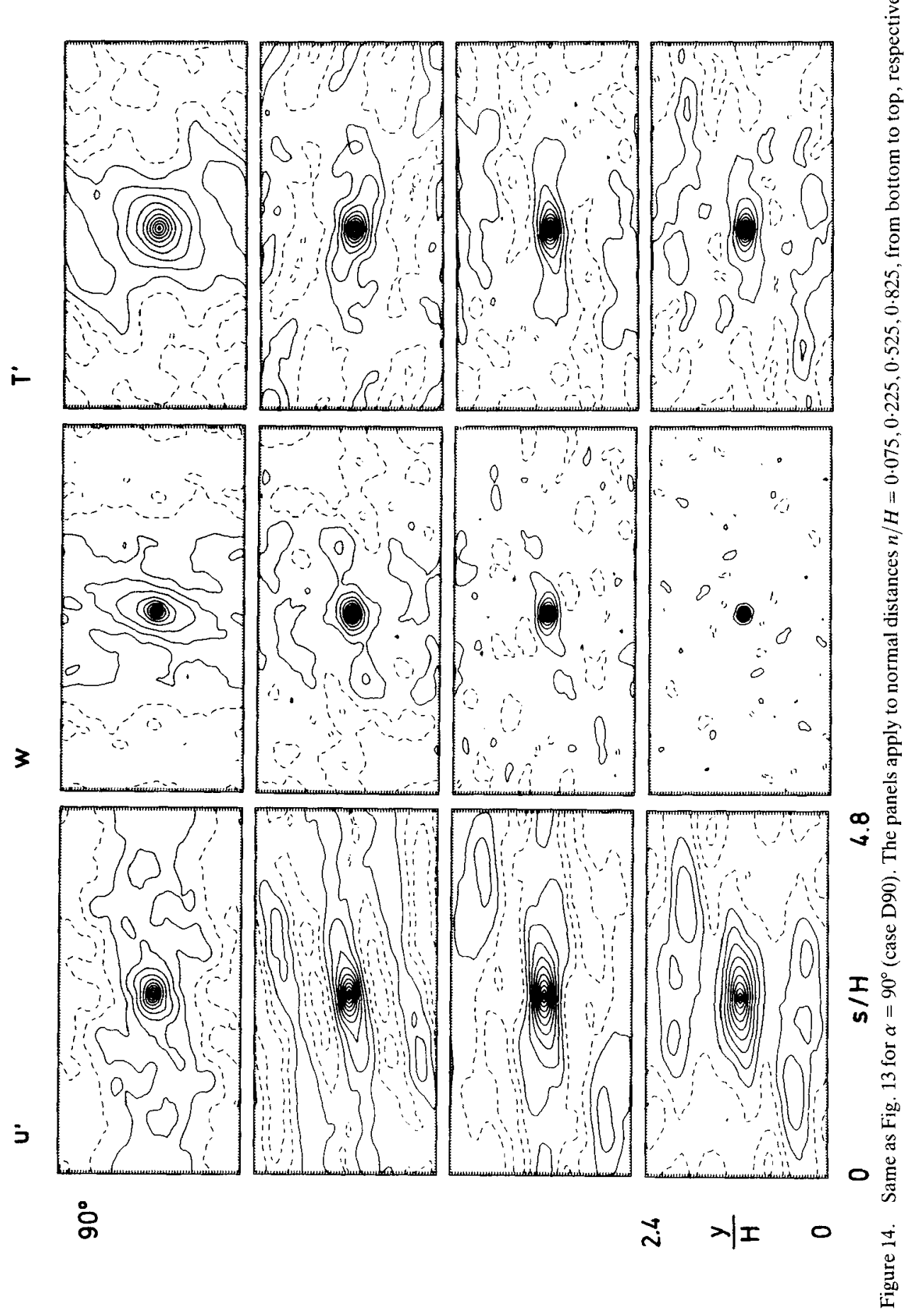


and negative components of $\vec{r}$. The correlations are computed just from the results for one instant of time, and therefore contain considerable but unknown statistical uncertainty at large separations $\vec{r}$.

The results support what we have learned from the instantaneous contour plots in quantitative sense, but also show strong variations with height. In any case, we observe that the correlations show a strong decrease over small distances which correspond to the integral length-scales of turbulence. The correlations at large separations signify large-scale coherent structures. Obviously, the present motion fields are a mixture of small-scale turbulence and large-scale coherent motions. The integral length-scales are very small near the surface; they are smallest for $w$, larger for $T$ and largest for $u$. The integral scales grow and the scales for the various fields become comparable in magnitude with increasing distance from the wall. The large-scale coherence indicates that the size of the computational domain is not totally sufficient to capture all relevant scales. It is difficult to quantify the effect of this limitation without further (expensive) simulations but there is no indication that these effects are large.

For $\alpha=10^{\circ}$ (Fig. 13) the large-scale structure appears to be formed mainly by rolls with axes in the cross-stream direction $y$. However, the picture is not very clear, because near the surface, the coherence in the $w$ component is small. At larger heights, both $w$ and $T$ show a pattern characteristic for such rolls but the $u$ component appears to be affected by rolls with an axis either in an up-slope or in a cross-slope direction. In contrast, for $\alpha=90^{\circ}$, Fig. 14 indicates large-scale rolls with axes parallel to the up-slope direction. Such rolls are typical for the inflection-point instability. At the outer edge of the boundary layer, the cross-stream correlation increases, in particular for $w$ and $T$ and this coherence is more typical for the Kelvin-Helmholtz instability.

Figure 15 depicts the same flow field as in Figs. 11 and 12 but in a plane normal to the up-slope direction. The $w$ and $v$ components are small and show little indications of roll structures if at all. For $\alpha=10^{\circ}$, the height of the mixed layer is rather uniform in the cross-stream direction $y$, and the temperature is more strongly correlated with $w$ than with $u$. For $\alpha=90^{\circ}$, the opposite is true. In any case, coherent structures are not dominant in this flow relative to the random turbulent motions.

For mixed convective/shear-driven turbulent boundary layers it is known that longitudinal rolls in the main stream direction form when $-d_{i} L_{*}^{-1}<5$ (Deardorff 1972), where $L_{*}$ is the Obukhov length. In the present units, the ratio equals $-d_{i} L_{*}^{-1}=$ $\kappa w_{*}^{3} u_{*}^{-3}=\kappa\left(d_{i} / H\right) /\left(u_{*} / v_{*}\right)^{3} ; \kappa=0.4$ is the von Karman constant. From the results of Table 2 we compute $-d_{i} L_{*}^{*-1}=30 \cdot 4,32 \cdot 7,30 \cdot 4,30 \cdot 6,25 \cdot 7,18 \cdot 6,8 \cdot 05,1 \cdot 65$ for $\alpha=2$, $4,7,10,20,30,45,90^{\circ}$, respectively. The values of the length-scale ratio suggest that longitudinal rolls should exist only for very steep slopes, which is consistent with the LES results.

\section{(e) Bulk properties versus slope-angle and roughness height}

Figure 16 depicts the variation of various characteristic values (as given in Table 2) versus $\sin \alpha$. The figure contains both the results from the coarse-grid cases Bxx and the highresolution cases Dxx. Generally, we find very small differences between the results from these cases. The largest differences occur for $\alpha=90^{\circ}$, as noted above. The full and dashed curves in Fig. 16 discriminate between two sets in which the roughness height $z_{0}$ differs by a factor of two. For $\alpha=10^{\circ}$, some results are also plotted, versus a wider range of roughness heights, in Fig. 17.

We observe rather weak dependence on the angle $\alpha$ for $\Delta T_{\mathrm{s}}, U_{\max }$ and $u_{*}$. However, one should note the logarithmic scales and that these quantities vary typically by a factor of two versus angle. Stronger variations are found for the boundary-layer thickness-scales 


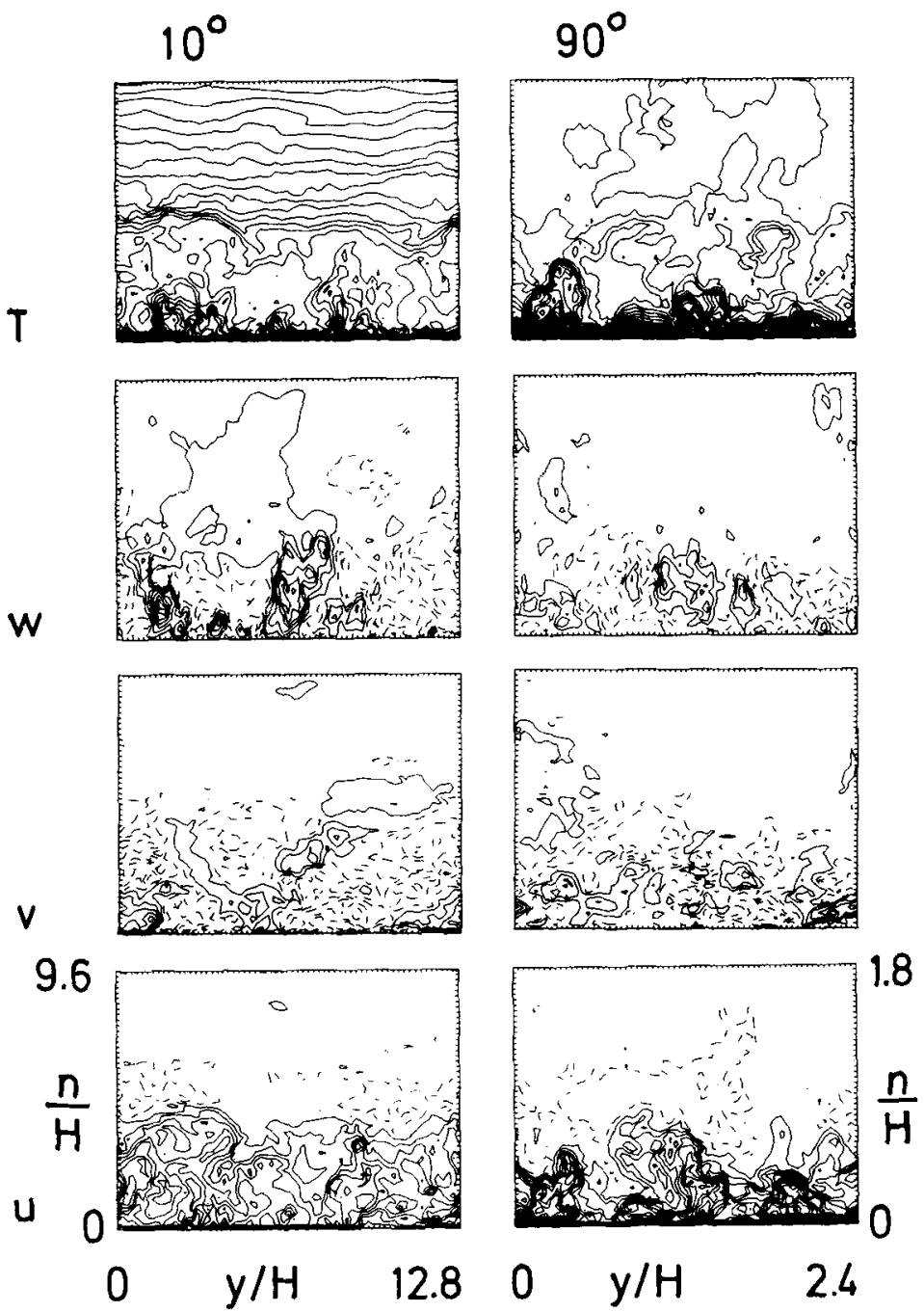

Figure 15. Instantaneous turbulent flow fields in an $n-y$ cross-section normal to the up-slope flow at $s=0$. The panels show from top to bottom: temperature $T+\theta$, and the velocity components $w, v$, and $u$. Left panels: $\alpha=10^{\circ}$; right panels: $\alpha=90^{\circ}$. All contour increments equal 0.5 in the appropriate units. Results from cases D10 and D90.

and for various integral quantities. Except for $U D$, which strictly follows $\sin ^{-1} \alpha$ according to Eq. (15), the results do not generally follow a power law $(\sin \alpha)^{x}$ with constant exponent $x$. Moreover, the results should not be extrapolated linearly to smaller angles because this would imply unrealistic behaviour like $d_{T}>d_{u}$, and $U_{\max } / w_{*} \rightarrow \infty$, where $w_{*} \sim d_{i}^{1 / 3}$.

The dependence on the roughness height $z_{0}$, Fig. 17, is generally weak and approximately linear in double-logarithmic scales. Figure 17 contains the exponents of proper power laws as determined from this graph. Of course, we cannot exclude different exponent values from arising at other slope-angles. Variables most sensitive to roughness are the friction velocity $u_{*}$, which grows when the surface gets rougher, and the temperature difference $\Delta T_{s}$, which gets reduced for better heat transfer on a rougher surface. The exponents of the corresponding power laws $(0.146,-0.307)$ are close to $(0 \cdot 166,-0.333)$ found in Schumann (1988) for corresponding results on a horizontal 


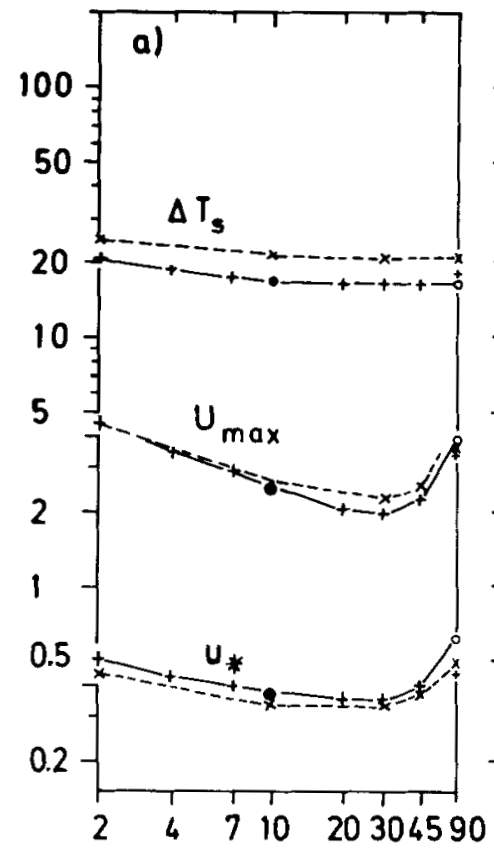

$\alpha$

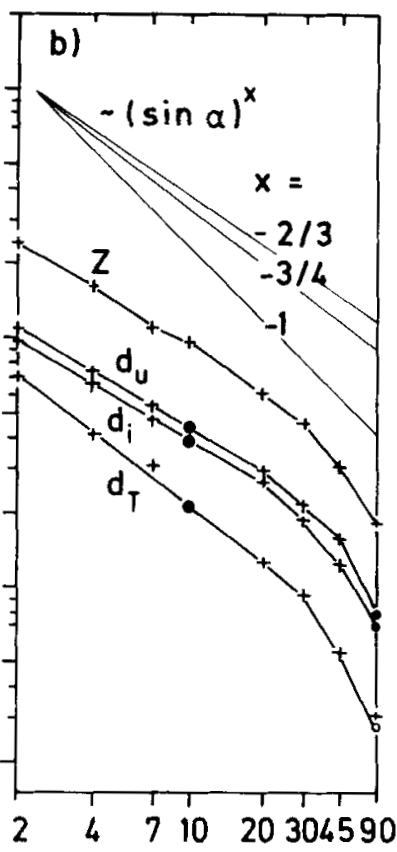

a

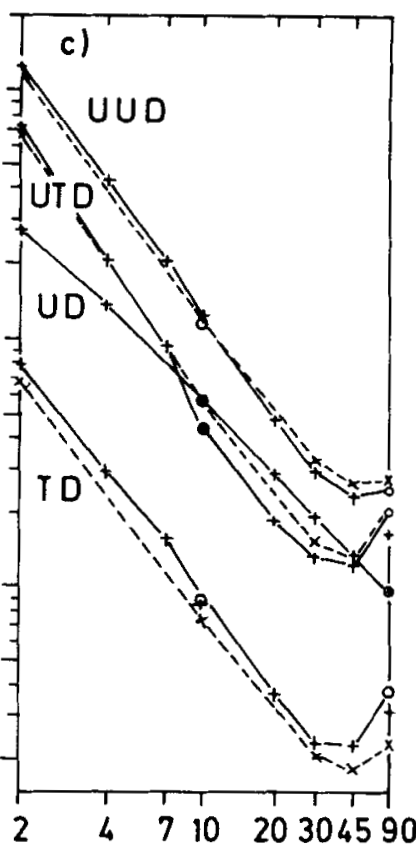

a

Figure 16. Mean values versus $\sin \alpha$ in double-logarithmic scales, + : results from cases B02 to B90 $\left(z_{0} / H=\right.$ $0.003) ; \mathrm{O}: \mathrm{D} 10, \mathrm{D} 90 ; \times: \mathrm{R} 02$ to $\mathrm{R} 90\left(z_{\mathrm{o}} / H=0.0015\right)$. In the middle panel, the thin straight lines indicate power laws, $\Delta T_{s}=$ temperature difference, $U_{\max }=$ maximum mean velocity, $u_{*}=$ friction velocity, $Z=$ 'height' of the computational domain, $d_{i}, d_{i}, d_{T}=$ boundary-layer thickness in terms of zero-velocity, minimum normal heat flux, zero-temperature, respectively. Integral values are $U U D=\int\langle u\rangle^{2} d n, U T D=\int\langle u\rangle\langle T\rangle d n, U D=$

$\int\langle u\rangle d n$, and $T D=\int\langle T\rangle d n$. All quantities are normalized in terms of $H, v_{*}$, or $\theta_{*}$.

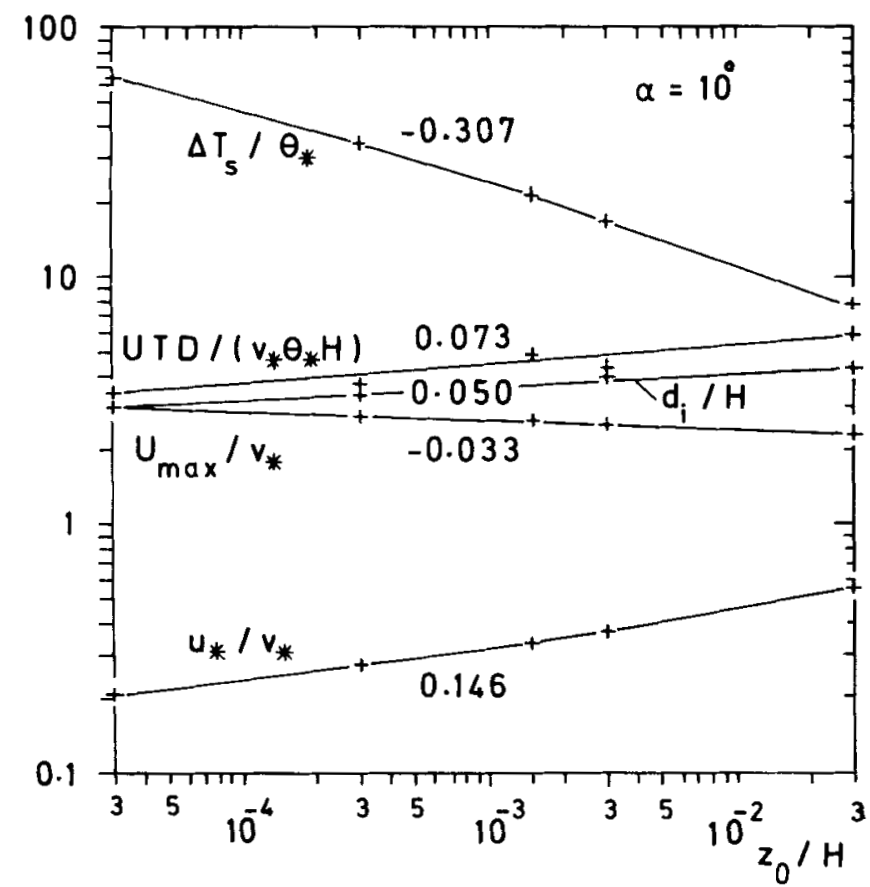

Figure 17. Mean values versus roughness height $z_{0}$, + : results from the series B10, R10, C10, E10 and F10, all for $\alpha=10^{\circ}$. Symbols and normalization as in Fig. 16. The numbers in the centre denote the exponent in power-law approximations to interpolating straight lines. 
surface. The small magnitude of the exponents for $U_{\max }$ and other internal parameters shows that turbulence inside the UBL is only slightly affected by surface friction.

For a subrange of angles and roughness parameter values, power laws may give reasonable approximations and are of practical interest. We have, therefore, set up power laws based on the numerical results given in Table 2 , in the range $2^{\circ} \leqslant \alpha \leqslant 30^{\circ}$. The coefficients $a, b$, and $c$ in the approximation $f=a\left(z_{0} / H\right)^{b}(\sin \alpha)^{c}$ for any quantity $f$ are determined so that the maximum deviation magnitude for all data is minimized. The numerical results are given in Table 3 together with the resultant standard and maximum deviations. The results for $U D$ and $T D$ in this table have been calculated according to Eqs. (14) and (15). The exponents $b$ differ slightly from those given in Fig. 17, because the latter are determined from the plots.

TABLE 3. COEFFICIENTS OF POWER-LAW APPROXIMATIONS

\begin{tabular}{|c|c|c|c|c|c|c|c|c|c|c|}
\hline $\begin{array}{l}\text { var. } \\
\text { unit }\end{array}$ & $\begin{array}{l}u_{*} \\
v_{*}\end{array}$ & $\begin{array}{c}U_{\max } \\
v_{*}\end{array}$ & $\begin{array}{c}\Delta T_{\mathrm{s}} \\
\theta_{*}\end{array}$ & $\begin{array}{l}d_{u} \\
H\end{array}$ & $\begin{array}{l}d_{i} \\
H\end{array}$ & $\begin{array}{l}d_{T} \\
H\end{array}$ & $\begin{array}{c}U D \\
v_{*} H\end{array}$ & $\begin{array}{c}T D \\
\theta_{*} H\end{array}$ & $\begin{array}{c}U T D \\
v_{*} \theta_{*} H\end{array}$ & $\begin{array}{c}U U D \\
v_{*}^{2} H\end{array}$ \\
\hline$a$ & 0.780 & 1.299 & $2 \cdot 684$ & 1.708 & 1.729 & 0.903 & 1 & 0.608 & 0.662 & 0.976 \\
\hline$b$ & 0.159 & -0.034 & -0.293 & 0.027 & 0.046 & 0.079 & 0 & 0.317 & $0 \cdot 074$ & -0.017 \\
\hline$c$ & -0.134 & $-0 \cdot 308$ & $-0 \cdot 078$ & $-0 \cdot 606$ & -0.604 & -0.754 & -1 & -1.268 & -1.507 & -1.407 \\
\hline$\sigma$ & 0.013 & 0.167 & 1.02 & 0.086 & 0.152 & 0.131 & 0.42 & 0.48 & 1.65 & 1.55 \\
\hline$\varepsilon$ & 0.018 & 0.257 & $2 \cdot 18$ & 0.137 & 0.361 & $0 \cdot 241$ & 0.98 & 1.22 & $3 \cdot 37$ & $3 \cdot 12$ \\
\hline
\end{tabular}

For each variable $f$, the expression $f=a\left(z_{0} / H\right)^{b}(\sin \alpha)^{c}$, where $a, b$, and $c$ are given above, approximates the results of Table 2 for $2^{\circ} \leq \alpha \leq 30^{\circ}, 3 \times 10^{-5} \leq z_{0} / H \leq 3 \times 10^{-2}$, in terms of its reference units with standard deviation $\sigma$ and maximum deviation $\varepsilon$.

For practical applications in remote sensing that relate surface temperature difference and heat flux, an interpolation has been set up which covers all angles so that

$$
\Delta T_{\mathrm{s}} / \theta_{*}=\mathrm{C}\left(\mathrm{H} / \mathrm{z}_{0}\right)^{0.286} \quad \mathrm{C}=3 \cdot 21+0 \cdot 122 \mathrm{x}+0 \cdot 089 \mathrm{x}^{2} \quad \mathrm{x}=\ln \sin \alpha
$$

approximates the LES-results for $2^{\circ} \leqslant \alpha \leqslant 90^{\circ}, 3 \times 10^{-5} \leqslant z_{0} / H \leqslant 3 \times 10^{-2}$, up to a standard deviation of 0.65 and maximum deviation $1 \cdot 21$. In terms of the independent external parameters this implies

$$
\Delta T_{\mathrm{s}}=C Q_{s}^{0.643}(\beta g)^{-0.357} z_{0}^{-0.286} N^{0.071} .
$$

It is interesting to note the very weak dependence on the Brunt-Väisälä frequency $N$ so that this parameter needs to be known only roughly. In fact, the dependence on the external parameters is only little different from that for the heat transfer in the convective boundary layer over a horizontal surface at zero mean wind-speed for which Schumann (1988) found $\Delta T_{\mathrm{s}}=10^{1 / 3} Q_{\mathrm{s}}^{2 / 3}(\beta g)^{-1 / 3} z_{0}^{-1 / 3}$. This relationship might be used for $\alpha<2^{\circ}$. If one wants to know the heat transfer as a function of the temperature difference, one can invert Eq. (22) to obtain

$$
Q_{\mathrm{s}}=\left(\Delta T_{s} / C\right)^{1.555} N^{-0.110}(\beta g)^{0.555} z_{0}^{0.445} .
$$

\section{COMPARISON WITH OTHER THEORIES}

(a) Estimates of the boundary-layer thickness

Prandtl's (1942) analytical solution predicts

$$
\begin{gathered}
d_{u}=2 d_{T}=\pi \delta \quad \delta=\left(\frac{4 \nu \mu}{N^{2} \sin ^{2} \alpha}\right)^{1 / 4} \\
U_{\max }=\frac{Q_{\mathrm{s}} N \delta}{\mu d \theta / d z}\left(\frac{\mu}{\nu}\right)^{1 / 2} \quad \Delta T_{\mathrm{s}}=Q_{\mathrm{s}} \delta / \mu
\end{gathered}
$$


i.e., $\delta \sim U_{\max } \sim \Delta T_{\mathrm{s}} \sim(\sin \alpha)^{-1 / 2}$ for specified heat flux and constant diffusivities $\nu$ and $\mu$ of momentum and heat, respectively. Obviously, the turbulent results differ strongly from these predictions. However, from the laminar solutions we may try to estimate the functional dependence of various bulk quantities on $\alpha$ by using proper approximations for the effective diffusivities.

For example, if we assume that both diffusivities are essentially due to shear and a mixing length of order $\delta$, i.e. $\nu \sim \mu \sim U \delta$, then Prandtl's solution results in

$$
\delta \sim H(\sin \alpha)^{-1} \quad U_{\max } \sim v_{*} \quad \Delta T_{\mathrm{s}} \sim \theta_{*} .
$$

This dependence of $\delta$ on $\sin \alpha$ is approximately observable in the LES results for large angles where shear contributes strongly to turbulence energy production. However, buoyancy forcing is still important at large angles. Hence, this estimate does not describe the increase of $U_{\max }$ and $\Delta T_{\mathrm{s}}$ with $\alpha$ at large angles, and therefore gives, at best, firstorder approximations for large slope-angles.

If we assume, on the other hand, that the diffusivities are induced mainly by convective motions, then we may apply the convective velocity scale to estimate $\nu \sim \mu \sim\left(\beta g Q_{\mathrm{s}} \delta\right)^{1 / 3} \delta$. This results in

$$
\delta \sim H(\sin \alpha)^{-3 / 2} \quad U_{\max } \sim v_{*}(\sin \alpha)^{1 / 2} \quad \Delta T_{\mathrm{s}} \sim \theta_{*}(\sin \alpha)^{1 / 2} .
$$

This prediction differs essentially from the LES-results, even for small angles, and cannot therefore provide an acceptable parametrization. The disagreement is a consequence of large-shear contributions to turbulence diffusivities even at small slope-angles.

As an alternative approach, we try to estimate the functional dependence on $\alpha$ not by using Prandtl's solution as a basis, but rather by referring to our observation of the well-mixed layer for angles $\alpha<20^{\circ}$, and by referring to the integral balances for steady state given in (14) and (15). Thus, we postulate that the temperature profile is well mixed, i.e. $d T / d z \cong-d \theta / d z$, over a layer of thickness $D \sim \delta$, so that $T D \cong \delta^{2} d \theta / d z$. Then, from Eq. (14), we estimate $u_{*}^{2}=N^{2} \delta^{2} \sin \alpha$. If we assume in addition that $U \sim u_{*}$, then Eq. (15) implies

$$
\delta \sim H(\sin \alpha)^{-3 / 4} \quad U_{\max } \sim v_{*}(\sin \alpha)^{-1 / 4} \quad \Delta T_{\mathrm{s}} \sim \theta_{*}(\sin \alpha)^{-3 / 4} .
$$

This model comes rather close to the LES-results for $d_{T}$ and $U_{\max }$ but overestimates the trend of $\Delta T_{\mathrm{s}}$ on $\alpha$ (presumably because it refers to $T$ above the surface in the mixed layer and not to the temperature at the surface itself). It implies that $u_{*} / w_{*}$ and $U_{\max } / w_{*}$ are constant for small angles because $w_{*} \sim \delta^{1 / 3}$. It is also reasonable that $\delta \sim d_{T}$ varies more strongly with $\alpha$ than $d_{u}$ or $d_{i}$, because the height of the mixed layer vanishes rather early for $\alpha \cong 10^{\circ}$. Hence, this model approximates the convective conditions at $\alpha \leqslant 10^{\circ}$ and presumably provides the correct tendencies for $\alpha \leqslant 2^{\circ}$.

\section{(b) Comparison with Brehm}

The most relevant study of comparable one-dimensional models has been presented by Brehm (1986). He has solved the one-dimensional ensemble-averaged equations of motion, for the same type of UBL as discussed in this paper, using a closure model which includes a transport equation for the turbulent kinetic energy and a prescribed lengthscale. He has determined steady-state solutions for a given temperature difference $\Delta T_{\mathrm{s}}$ and for several parameter values including slope-angles between $2^{\circ}$ and $30^{\circ}$. The mean profiles differ qualitatively from those shown in Fig. 3, in that Brehm's results do not show the strong return flow for small slope-angles.

The most uncertain part of such a model is the prescribed length-scale. For example, Brehm (1986) follows Mellor and Yamada (1982), and computes the dissipation from 
$\langle\varepsilon\rangle=\langle E\rangle^{3 / 2} / L$, where $L=\ell_{\mathrm{m}} / 0 \cdot 2^{3 / 2}$ is a dissipation length-scale which uses a mixing length $\ell_{\mathrm{m}}$. The latter is determined as a function of normal distance, a local Richardson number and the profile of kinetic energy. The result for $\alpha=10^{\circ}$ has been taken from Fig. 4.8 of Brehm (1986) and is shown together with the LES results in Fig. 18. The statistical uncertainty of the LES results amounts to about $\pm 0 \cdot 1$. The results agree with those for the convective boundary layer over horizontal planes as reported by Krettenauer and Schumann (1989) and Bougeault and Lacarrère (1989). We find that the model overestimates $L$ inside the UBL with the consequence that it also over-estimates the kinetic energy for given energy production and dissipation. As pointed out to me by one of the reviewers, outside the boundary layer the reason for the large discrepancy between the LES dissipation scale and Brehm's is probably the presence of gravity waves in the LES. The waves contain fluctuation energy but do not produce dissipation, and consequently give a large effective dissipation scale. The one-dimensional model does not contain waves, and only considers the random turbulent motions which cascade energy to smaller scales. As shown by Fig. 5, the waves contribute to momentum transfer but the divergence of the flux is small and, hence, they affect the momentum balance only little. Moreover, Brehm's model assumes down-gradient momentum transport whereas the LES-results imply deviations from down-gradient transport near the outer edge of the boundary layer at small angles. Hence, it is not surprising that Brehm's model results in different mean velocity profiles.

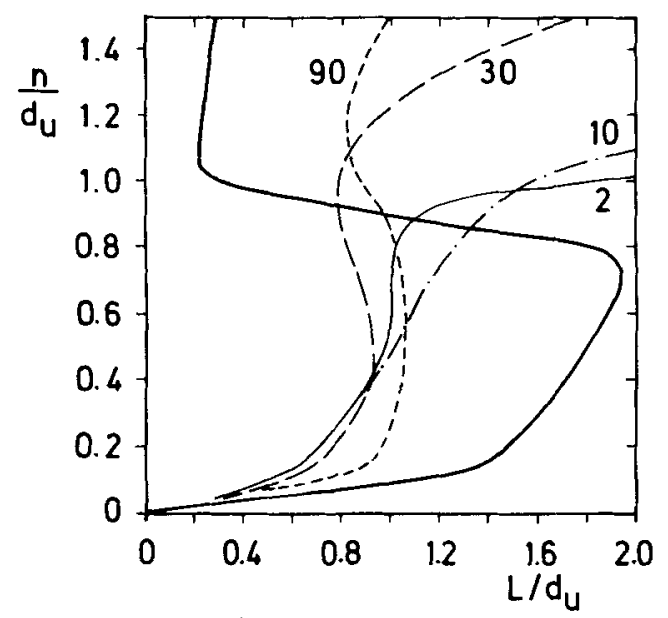

Figure 18. Dissipation length-scale $L=\langle E\rangle^{3 / 2} /\langle\varepsilon\rangle$ normalized by the velocity-boundary-layer thickness $d_{u}$ versus normal distance $n$. The thick curve is taken from Brehm (1986) for $\alpha=10^{\circ}$. The other curves depict the LES-results for $\alpha=2,10,30$ and $90^{\circ}$, line-coding as in Fig. 4.

Brehm expressed the bulk properties of his simulations non-dimensionally in terms of friction coefficients $c_{\mathrm{g}}$ for momentum and $c_{\mathrm{H}}$ for heat transfer, which he defined as

$$
c_{\mathrm{g}}=u_{*} /\left(\kappa \Delta T_{\mathrm{s}} \beta g / N\right) \quad c_{\mathrm{H}}=-Q_{\mathrm{s}} /\left(\kappa u_{*} \Delta T_{\mathrm{s}}\right) \text {. }
$$

Brehm uses $K=0.36$ as the value of the von Karman constant. He shows that $c_{\mathrm{g}}$ and $c_{\mathrm{H}}$ depend solely on the angle $\alpha$ and an 'analogous Rossby number'

$$
R o=\frac{\kappa \Delta T_{\mathrm{s}}}{z_{0}(d \theta / d z) \sin \alpha}=\frac{\kappa}{\sin \alpha} \frac{\Delta T_{\mathrm{s}}}{\theta_{*}} \frac{H}{z_{0}}
$$

The results $c_{\mathrm{g}}(R o, \alpha)$ and $\eta(R o, \alpha)=c_{\mathrm{H}} / c_{\mathrm{g}}$ are given in Fig. 5.3 of Brehm (1986). They have been converted into a table with proper interpolating subroutines by $\mathrm{H}$. Mannstein 
(1989, personal communication). Based on these subroutines, we evaluate

$$
\Delta T_{\mathrm{s}} / \theta_{*}=\left[K c_{\mathrm{g}}\{-\eta(R o, \alpha)\}^{1 / 2}\right]^{-1} .
$$

This requires iterations because $R o$ itself depends on $\Delta T_{\mathrm{s}}$ see Eq. (29). Thereafter, we obtain

and

$$
u_{*} / v_{*}=c_{\mathrm{g}}(R o, \alpha) \kappa \Delta T_{\mathrm{s}} H /\left(\theta_{*} z_{0}\right)
$$

$$
H_{*} / H=u_{*} /\left(v_{*} \sin \alpha\right)
$$

The latter result is then used to evaluate UTD from Fig. 5.7 of Brehm (1986) (for $\alpha=$ $2,10,20$ and $30^{\circ}$ ). The results for some selected cases are compared in Table 4 with the results from the LES.

The differences in $u_{*}$ range from $3 \%$ to $50 \%$ of the LES results. The differences for the temperature difference $\Delta T_{\mathrm{s}}$ are generally smaller and typically about $10 \%$. The results for UTD show very large differences (up to $90 \%$ ). This is not surprising in view of the difficulty of modelling the thickness of the boundary layer and the profiles of mean

\begin{tabular}{|c|c|c|c|}
\hline $\begin{array}{l}\text { var. } \\
\text { unit }\end{array}$ & $\begin{array}{l}u_{*} \\
v_{*}\end{array}$ & $\begin{array}{c}\Delta T_{i} \\
\theta_{*}\end{array}$ & $\begin{array}{c}U T D \\
v_{*} \theta_{*} H\end{array}$ \\
\hline \multirow[t]{2}{*}{ B 02} & 0.503 & $20 \cdot 43$ & 71 \\
\hline & 0.515 & $22 \cdot 23$ & 58 \\
\hline \multirow[t]{2}{*}{ B04 } & 0.43 & $18 \cdot 51$ & \\
\hline & 0.502 & $20 \cdot 15$ & \\
\hline \multirow[t]{2}{*}{ B 07} & 0.397 & $17 \cdot 39$ & \\
\hline & 0.473 & $19 \cdot 64$ & \\
\hline \multirow[t]{2}{*}{$\mathrm{B} 10$} & $0 \cdot 371$ & $16 \cdot 75$ & 4.37 \\
\hline & 0.472 & $19 \cdot 86$ & $7 \cdot 3$ \\
\hline \multirow[t]{2}{*}{$\mathrm{B} 20$} & 0.344 & $16 \cdot 14$ & $1 \cdot 88$ \\
\hline & 0.497 & 17.72 & $3 \cdot 6$ \\
\hline \multirow[t]{2}{*}{ B30 } & 0.343 & $16 \cdot 11$ & $1 \cdot 32$ \\
\hline & 0.521 & $18 \cdot 24$ & $2 \cdot 4$ \\
\hline \multirow[t]{2}{*}{$\mathrm{C} 10$} & 0.568 & $7 \cdot 694$ & $5 \cdot 84$ \\
\hline & $(3.649$ & $10 \cdot 27$ & $7 \cdot 1$ \\
\hline \multirow[t]{2}{*}{ E10 } & 0.266 & 34.44 & 3.87 \\
\hline & 0.371 & $32 \cdot 38$ & $7 \cdot 3$ \\
\hline
\end{tabular}
temperature and velocity. In conclusion, both methods give results of the same order of magnitude but with essential differences in detail.

(c) Relationship between bulk quantities

For closure of the bulk equations, given in Eqs. (14) and (15), for non-steady boundary layers, one requires relationships between the unknown quantities $U U D, U T D$, $W D, u_{*}^{2}$, and those quantities which can be predicted from these equations, i.e. $U D$ and $T D$. In the present simulations, $W D \equiv 0$ because of the boundary conditions selected, and therefore, we cannot comment on the various proposals for its dependence on other field quantities, as discussed by Egger (1990). To some extent the negative heat flux at the outer edge of the boundary layer is a measure for entrainment, and Fig. 7 shows that this type of entrainment is large only for $\alpha \leqslant 10^{\circ}$. However, the other terms have been evaluated so that we can check existing closure assumptions. For example, Brehm (1986), as also discussed by Egger (1990), has proposed relating $U D$ to $U T D^{2 / 3}$. This proposal 
is not very appealing because it requires further factors for dimensional reasons. A parametrization of the form

$$
U T D=c\left(\alpha, z_{0} / H\right) U D \cdot T D / d_{u}
$$

where the coefficient $c$ and the thickness $d_{u}$ are to be prescribed as functions of the external parameters, appears to be more consistent in this sense. However, as shown in Fig. 19, Brehm's (1986) proposal fits the tendency of the LES-results rather well, at least for $\alpha<30^{\circ}$. It is an easy task to determine the quantitative relationship between UTD and $U D$ or other bulk quantities using the power laws given in Table 3 .

We can also check the parametrization

$$
U_{\max }^{2}=C \beta g T D \sin \alpha
$$

proposed by Vergeiner (1982) (It is not quite clear, however, whether the symbol $V$ in Vergeiner's paper refers to $U_{\text {max }}$ or a suitable mean velocity like $U D / d_{u}$ ). Vergeiner assumes that $C$ corresponds to a friction coefficient of about unity. From the power laws given in Table 3, we find

$$
C=2 \cdot 77\left(z_{0} / H\right)^{-0 \cdot 38}(\sin \alpha)^{-0 \cdot 35} .
$$

For $\alpha=10^{\circ}, z_{0} / H=0 \cdot 003$, it amounts to $C=46 \cdot 5$. Thus, the coefficient is quite different from unity and varies rather strongly with $\alpha$, even in the range of validity of these power laws, i.e. for $2^{\circ} \leqslant \alpha \leqslant 30^{\circ}$. Using $U D / d_{u}$ instead of $U_{\max }$ gives a similar result.

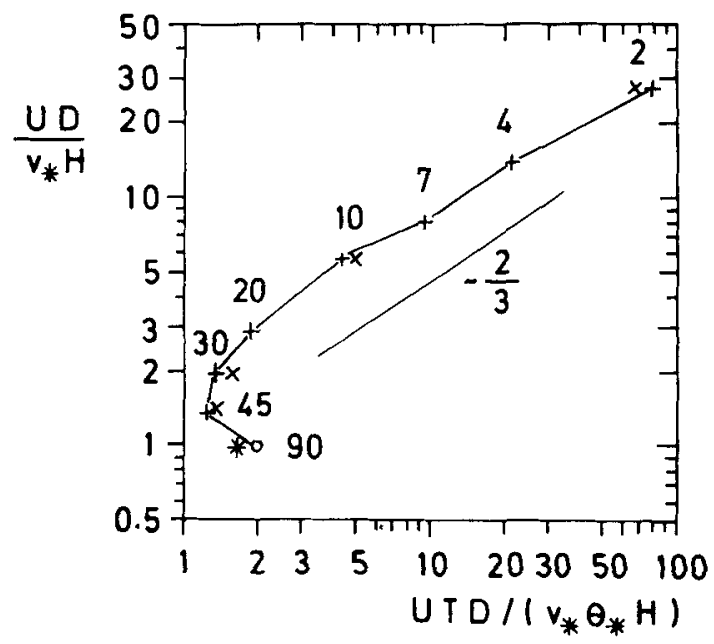

Figure 19. Integrated mass flux versus integrated up-slope heat flux. The symbols have the meaning as in Fig. 16. The numbers denote the size of the angle. The thin line corresponds to a power law with exponent $2 / 3$.

\section{SUMMARY AND CONCLUSIONS}

By LES we have determined the structure of the quasi-steady boundary layer which forms at the interface between a stratified atmosphere at rest and a uniformly rough and heated inclined or vertical plane surface. The steady-state solutions depend solely on the angle $\alpha$ and the length-scale ratio $z_{0} / H$. The dependence on surface heat flux, stratification and buoyancy coefficients is described by the scaling parameters given in Eq. (1). We have shown that the results are only slightly sensitive to grid variations of the LES so that the results may be treated like experimental measurements. In fact, none of the existing measurements give such a complete picture as the LES. Some uncertainty arises 
from application of the Monin-Obukhov boundary conditions which we cannot quantify. In particular, the concept of surface roughness is an artifact which limits any comparison with real conditions. However, the effect of friction on the internal structure of the turbulent boundary layer is rather weak so that the results do not depend strongly on the boundary condition.

It takes several oscillation periods $2 \pi /(N \sin \alpha)$ to reach a steady state, so that a stationary UBL can be expected to exist at mountain slopes only for large angles. This time scale is independent of diffusivity and the layer's thickness but applies only to onedimensional motions which are invariant relative to the up-slope coordinate. As shown by Egger (1981), small scale circulations and the flow near the surface have smaller timescales of the order $N^{-1}$. This explains observations of instantaneous local start-up of upslope motion at sunrise as mentioned by Vergeiner and Dreiseitl (1987). Note that the steady-state solution for infinitesimally small angles is basically different from the solution for the growing convective boundary layer for zero slope-angle. The subject of nonsteady growing UBL at small angles deserves further investigation in the future.

For $\alpha \leqslant 10^{\circ}$, a well-mixed layer is formed which causes a strong temperature inversion and strong down-slope flow at the outer edge of the UBL. Deviations from down-gradient momentum transport (negative effective viscosity) seem to be connected with the strong return flow at small angles. For all angles, turbulence is generated by both shear and buoyancy. At small angles, buoyancy effects contribute about $60 \%$. This contribution decreases for angles larger than $20^{\circ}$ and shear contributes most $(70 \%)$ to turbulence energy for $\alpha=90^{\circ}$. The comparison with the data of Deardorff and Willis (1987) for $\alpha=10^{\circ}$, suffers from advection effects in the non-uniform laboratory slope layer but these authors also note the large contributions from shear to turbulence generation. The LES results for the UBL at small angles agree closely with measurements on variances, turbulence energy budget and length-scales reported by Zhou et al. (1985) for a baroclinic convective boundary layer.

Some effort has been made to identify coherent structures in the turbulent boundary layers. In agreement with the observations of Deardorff and Willis (1987), the instantaneous flow fields and the correlation functions exhibit contributions from all scales in a random manner with only small contributions from coherent large-scale motions. At small angles, we find some indication of cross-slope rolls or Kelvin-Helmholtz waves. Such rolls are rather unexpected from linear theories and not yet fully explained. The normal velocity fluctuations have a skewed distribution and transport kinetic energy towards the outer edge of the UBL. Pressure diffusion continues to transport energy away from the UBL into the stratified atmosphere. The polygonal spoke pattern, which was observed for convection over horizontal surfaces (Schmidt and Schumann 1989), appears to be destroyed by the shear on inclined surfaces. At steep slopes, hot fluid parcels have largest up-slope velocity. Near the wall, longitudinal rolls parallel to the mean flow can be identified while Kelvin-Helmholtz-type waves are prevailing at the outer edge of the UBL. The present results are consistent with the requirement of large Obukhov length for longitudinal rolls in mixed convective/shear-driven boundary layers.

We have quantitatively determined the dependence of several mean properties on slope angle and surface roughness. The results are difficult to explain with simple models. A model based on shear-induced diffusivities explains the tendencies best at large angles, while a model which assumes the existence of a well-mixed layer gives reasonable results at small angles, presumably including values $\alpha<2^{\circ}$. The dependence of surface temperature difference on surface roughness is not much different from that found for convection over a horizontal surface by Schumann (1988). For $2^{\circ} \leqslant \alpha \leqslant 30^{\circ}$, we have deduced power-law approximations for all mean quantities, and these approximations 
will be of some practical value. For the relationship between surface temperature difference and heat flux, a parametrization has been proposed which covers all angles $\alpha \geqslant 2^{\circ}$. Note that $\Delta T_{\mathrm{s}}$ refers to the surface temperature at $n=z_{0}$; this value is not well defined in reality and, hence, might be much different from a temperature difference measurable by remote sensing. Our temperature-difference results differ quantitatively by about $10 \%$ from those of Brehm (1986) who employed a one-dimensional turbulence model.

The present study assumes prescribed heat flux. This case is equivalent to that of prescribed surface temperature difference only in the steady laminar case. In turbulent flows, the different boundary conditions may cause different solutions because the heat flux varies as a function of the local motion field for prescribed temperature difference and this may cause a more intermittent flow structure. However, for the horizontal convective boundary layer, Krettenauer and Schumann (1989) have compared the results of LES for the different kinds of boundary conditions and found no significant difference. It might be that the difference is largest for down-slope motions on cooled surfaces because of the self-isolating effect of stable stratification. But for the day-time flow over dry surfaces, a prescription of heat flux seems to be more appropriate to nature because most of the solar energy will be converted into a flux of sensible heat, at least if the thermal inertia of the surface is small.

Atkinson, B. W.

Bougeault, $P$.

Bougeault, P. and Lacarrère, P.

Brehm, M.

Brown, R. A.

Clever, R. M. and Busse, F. H.

Deardorff, J. W

Deardorff, J. W. and Willis, G. E.

Defant, F.

Egger, J.

Gill, A. E.

Gill, A. E. and Davey, A. Hennemuth, B. and Schmidt, $H$.

Horst, T. W. and Doran, J. C.

\section{REFERENCES}

1981

1983

1989

1986

1980

1977

1972

1987

1981

1990

1966

1969

1985

1988 don
Meso-scale Atmospheric Circulations. Academic Press, Lon-

A non-reflective upper boundary condition for limited-height hydrostatic models. Mon. Weather Rev., 111, 420-429

Parameterization of orography-induced turbulence in a mesoscale-beta model. ibid., 117, 1872-1890

'Experimentelle und numerische Untersuchungen der Hangwindschicht und ihrer Rolle bei der Erwärmung von Tälern'. PhD-thesis, Universität München, Meteorologisches Institut, Wiss. Mitt. Nr. 54 (available on request from Meteorol. Inst., Univ. München, Theresienstr. 37, D-8000 München 2)

Longitudinal instabilities and secondary flows in the planetary boundary layer: A review. Rev. Geophys. Space Phys., 18, 683-697

Instabilities of longitudinal convection rolls in an inclined layer. J. Fluid Mech., 81, 107-127

Numerical investigation of neutral and unstable planetary boundary layers. J. Atmos. Sci., 29, 91-115

Turbulence within a baroclinic laboratory mixed layer above a sloping surface. ibid., 44, 772-778

Zur Theorie der Hangwinde, nebst Bemerkungen zur Theorie der Berg- und Talwinde. Arch. Met. Geophys. Biokl., Al, 421-450

On the linear two-dimensional theory of thermally induced slope winds. Beitr. Phys. Atmos., 54, 465-481

'Thermally forced flows: theory'. In Current directions in atmospheric processes over complex terrain, Meteorol. Mono., Am. Meteorol. Soc. (in press)

The boundary layer regime for convection in a rectangular cavity. J. Fluid Mech., 26, 515-536

Instabilities of a buoyancy-driven system. ibid., 35, 775-798

Wind phenomena in the Dischma valley during DISKUS. Arch. Met. Geoph. Biocl., Ser. B, 35, 361-387

The turbulence structure of nocturnal slope flow. J. Atmos. Sci., 45, 605-616 
Klemp, J. B. and Durran, D. R.

Kondo, J. and Sato, T.

Krettenauer, K. and Schumann, U. 1989

Launder, B. E.

Lykosov, V, N. and Gutman, L. N. 1972

Manins, P. C. and Sawford, B. L. Mannstein, $\mathrm{H}$.

Mason, P. J.

Mellor, G. L. and Yamada, T.

Prandtl, L.

Rao, K. S. and Snodgrass, H. F.

Schmidt, H. and Schumann, U.

Schumann, U.

Schumann, U., Hauf, T., Höller, H., Schmidt, H. and Volkert, $\mathrm{H}$.

Smolarkiewicz, P. K.

Turner, J. S.

Vergeiner, I.

Vergeiner, I. and Dreiseitl, E.

Veronis, G.

Whiteman, C. D.

Yamada, T. and Bunker, S.

Zhou, M. Y., Lenschow, D. H., Stankov, B. B., Kaimal, J. C. and Gaynor, J. E.

1988
An upper boundary condition permitting internal gravity wave radiation in numerical mesoscale models. Mon. Weather Rev., 111, 430-444

A simple model of drainage flow on a slope. Boundary-Layer Meteorol., 43, 103-123

Struktur der konvektiven Grenzschicht bei verschiedenen thermischen Randbedingungen. Deutscher Wetterdienst, Offenbach. Ann. Meteorol., 26, 278-279

On the effects of a gravitational field on the turbulent transport of heat and momentum. J. Fluid Mech., 67, 569-581

Turbulent boundary layer above a sloping underlying surface. transl. from Izv. Acad. Sci. USSR, in Atmos. Ocean. Phys., 8, 799-809

A model of katabatic winds. J. Atmos. Sci., 36, 619-630

'Die radiometrisch bestimmte Oberflächentemperatur im Gebirge und die Ermittlung des Stroms fühlbarer Wärme'. Thesis, DLR-FB-90-00, Institut für Physik der Atmosphäre, 8031 Oberpfaffenhofen, Germany

Large-eddy simulation of a convective atmospheric boundary layer. J. Atmos. Sci., 46, 1492-1516

Development of a turbulence closure model for geophysical fluid problems. Rev. Geophys. Space Phys., 20, 851-875

1942 Strömungslehre. Vieweg, Braunschweig, pp. 396-399

1981 A non-stationary nocturnal drainage flow model. Bound.Layer Meteorol., 20, 309-320

1989 Coherent structure of the convective boundary layer derived from large-eddy simulations. $J$. Fluid Mech., 200, 511562

Minimum friction velocity and heat transfer in the rough surface layer of a convective boundary layer. BoundaryLayer Meteorol., 44, 311-326

A mesoscale model for the simulation of turbulence, clouds and flow over mountains: Formulation and validation examples. Beitr. Phys. Atmos., 60, 413-446

A fully multidimensional positive definite advection transport algorithm with small implicit diffusion. J. Comput. Phys., 54, 325-362

Buoyancy effects in fluids. Cambridge, Univ. Press

Eine energetische Theorie der Hangwinde. Deutscher Wetterdienst, Offenbach. Ann. Meteorol. 19, 189-191

1987 Valley winds and slope winds-observations and elementary thoughts. Meteorol. Atmos. Phys., 36, 264-286

Analogous behaviour of homogeneous, rotating fluids and stratified, non-rotating fluids. Tellus, 19, 326-336

'Observations of thermally developed wind systems in mountainous terrain. In Current directions in atmospheric processes over complex terrain. Meteorol. Mono., Am. Meteorol. Soc. (in press)

Development of a nested grid, second moment turbulence closure model and application to the 1982 ASCOT brush creek data simulation. J. Appl. Meteorol., 27, 562-578

Wave and turbulence structure in a shallow baroclinic convective boundary layer and overlying inversion. J. Atmos. Sci. , 42, 47-57 